\title{
Nanocarriers-Mediated Drug Delivery Systems for Anticancer Agents: An Overview and Perspectives
}

This article was published in the following Dove Press journal: International Journal of Nanomedicine

\author{
Zehra Edis (D) ${ }^{1,2, *}$ \\ Junli Wang ${ }^{3, *}$ \\ Muhammad Khurram Waqas ${ }^{4}$ \\ Muhammad ljaz ${ }^{5}$ \\ Munazza ljaz \\ 'Department of Pharmaceutical Sciences, \\ College of Pharmacy and Health Sciences, \\ Ajman University, Ajman, United Arab \\ Emirates; ${ }^{2}$ Centre of Medical and Bio- \\ allied Health Sciences Research, Ajman \\ University, Ajman, United Arab Emirates; \\ ${ }^{3}$ Laboratory of Reproduction and \\ Genetics, Affiliated Hospital of Youjiang \\ Medical College for Nationalities, Baise, \\ Guangxi, People's Republic of China; \\ ${ }^{4}$ Institute of Pharmaceutical Sciences, \\ University of Veterinary and Animal \\ Sciences, Lahore, Pakistan; ${ }^{5}$ Department \\ of Pharmacy, COMSATS University \\ Islamabad, Lahore Campus, Lahore, \\ Pakistan; ' Institute of Molecular Biology \\ and Biotechnology, The University of \\ Lahore, Defense Road Campus, Lahore, \\ Pakistan
}

*These authors contributed equally to this work
Correspondence: Munazza ljaz Institute of Molecular Biology and Biotechnology, The University of Lahore, Defense Road Campus, Lahore, 54000, Pakistan

Email munazza3murtaza@gmail.com

\begin{abstract}
Nanotechnology has been actively integrated as drug carriers over the last few years to treat various cancers. The main hurdle in the clinical management of cancer is the development of multidrug resistance against chemotherapeutic agents. To overcome the limitations of chemotherapy, the researchers have been developing technological advances for significant progress in the oncotherapy by enabling the delivery of chemotherapeutic agents at increased drug content levels to the targeted spots. Several nano-drug delivery systems designed for tumor-targeting are evaluated in preclinical and clinical trials and showed promising outcomes in cancerous tumors' clinical management. This review describes nanocarrier's importance in managing different types of cancers and emphasizing nanocarriers for drug delivery and cancer nanotherapeutics. It also highlights the recent advances in nanocarriers-based delivery systems, including polymeric nanocarriers, micelles, nanotubes, dendrimers, magnetic nanoparticles, solid lipid nanoparticles, and quantum dots (QDs). The nanocarrier-based composites are discussed in terms of their structure, characteristics, and therapeutic applications in oncology. To conclude, the challenges and future exploration opportunities of nanocarriers in chemotherapeutics are also presented.
\end{abstract}

Keywords: nanomaterials, anticancer agents, recent advances, multidrug resistance, chemotherapy

\section{Overview}

Cancer is a group of disorders causing abnormal cell growth and can spread to other body parts. Globally, cancer-associated death rates come second after the deaths related to cardiovascular system diseases and are considered the most critical global health issue. Due to this prevalence, cancer therapy has become a joint dialogue among health-care providers and researchers. ${ }^{1}$ As stated by IARC (International Agency for Research on Cancer) worldwide, a total of $14.1 \mathrm{M}$ cases were reported in 2012, and $82 \%$ ( 8 million) of those cases were reported from underdeveloped countries. This geological difference in cancer frequency can be explained by many aspects, including age factor, number of screening tests, early diagnosis, and accessibility of quality treatment, and occurrence of risk factors. Similarly, according to the world health organization (WHO), the number of cancer patients will increase up to $22 \mathrm{M}$ within the next twenty years. ${ }^{2}$

Due to the massive impact of this deadly disease, researchers worldwide are focused on developing different novel carrier systems to deliver anticancerous agents at the target sites without producing harmful effects on healthy tissues. 
The outcome of therapy is determined by the nature of cancer, the malignant tumor area, and its progression stage. Different strategies, including immunotherapy, hormone therapy, surgery, chemotherapy, and radiotherapy, are used to treat cancer. Among these, chemotherapy is considered the prime choice for the eradication of cancerous cells. However, the conventional delivery systems for chemotherapy have some limitations, including toxic side effects of the chemotherapeutic agents on fast-growing healthy cells and other side effects like nausea, vomiting, fatigue, hair loss, and even death in extreme cases. Another main obstacle in the management of tumors is the development of multidrug resistance (MDR). The cancerous cell develops resistance and escapes from the effects of chemotherapeutic drugs. ${ }^{3-6}$ To overcome chemotherapy's limitations, the researchers are developing new nanotechnology-based advances in drug delivery systems for significant developments in oncotherapy by delivering anticancer agents at higher concentrations to targeted sites. ${ }^{7,8}$ Nanocarriers have been emerged and exploited as a new tool for cancer treatment to reduce numerous precincts of conventional drug delivery systems. ${ }^{9}$ Researchers are enthusiastically working to improve the treatment options for the management of malignant tumors. They have trialed nanotechnologybased drug carriers as useful cancer management applications, which further lead to nano-drug carriers (10-100 $\mathrm{nm}$ ) impending as a distinct therapeutics for cancer treatment. The potential use and efficacy of various nano-drug carriers for anticancer agents are much higher than normal ones. ${ }^{10}$ Nanocarriers offer multiple benefits over conventional drug delivery systems like increased plasma halflife, improved biodistribution, and targeted delivery of a drug to tumor microenvironment through endothelial layers. These nanocarrier-based drug delivery systems are being used against various types of cancerous tumors containing tissues.

Various nanocarrier preparations have been designed to improve therapeutic efficacy and reduce toxic side effects. Nanocarriers prospect a continuous, direct, and controlled drug release to the malignant cells selectively with augmented drug localization and cellular uptake. Nanoparticles can be automated to identify malignant cells and give discriminating and precise drug transfer, evading contact through the normal cells. Additionally, various drawbacks, including systemic toxicity, low oral bioavailability, decreased solubility, narrow therapeutic indices, and chemoresistance, have been overcome with the advancement of nanocarrier-based DDS. ${ }^{11-13}$

Nanotechnology has become a vast field for biomedical applications and nanocarriers such as polymeric nanoparticles, micelles, dendrimers, solid lipid nanoparticles, quantum dots, and magnetic nanoparticles with substantial physical and chemical properties along with the nanosized effect. ${ }^{14}$ The outcomes of various cancer research studies have generally acknowledged that nanoparticlebased drug delivery systems (DDS) with boosted bioavailability and minimum side effects embrace auspicious anticancer effects compared to free drugs. ${ }^{15-17}$

Different types of nanocarriers have been successfully adopted in various nanomedicine. Nevertheless, their transformation to medical oncology remains inadequate. Engineered nanoparticles possess advanced applications that include gene delivery, ${ }^{18}$ site-specific targeted drug delivery system, ${ }^{19}$ as well as agents in magnetic resonance imaging to enhance diagnostic possibilities, ${ }^{20}$ or to design and develop new imaging methods. ${ }^{21}$ Nanocarriers possess distinctive properties such as quantum effects, a high ratio of surface to volume, and an ability to carry therapeutically active compounds to the targeted site due to their nano size. ${ }^{22}$ This review focuses on polymeric nanoparticles, having unique identifying properties incorporated by various strategies that distinguish them from previous anticancer drug delivery systems. It also describes nanoparticles inside the cells, illustrating many successful research pieces about using different approaches to remove the side effects of conventional therapies with tailored cancer treatment.

Consequently, the current writing piece is about nanotechnology developments, concentrating on nanocarriers for anticancer drug delivery. It also describes the differences in the polymeric nanoparticles, micelles, dendrimers, solid lipid nanocarrier, quantum dots, and magnetic nanocarriers. Finally, the current challenges and future perspectives have been highlighted.

\section{Precincts of Conventional Drug Delivery Systems}

The delivery of chemical antineoplastic agents used as chemotherapy for local and metastatic tumors is associated with many drawbacks. The major problem related to chemotherapy is the lack of selectivity of the malignant cells; these chemotherapeutic agents have similar cytotoxic effects on the normal healthy cells. Therefore, the long- 
run clinical use of such chemotherapeutic agents causes severe toxicity to normal healthy cells. Chemotherapeutics delivered via the conventional delivery systems can induce severe cytotoxic effects to healthy cells due to the high volume of distribution of the drugs, which are low molecular weight and are cleared quickly. ${ }^{23}$

The chemicals with low therapeutic index administered in high concentration due to low bioavailability or early excretion from the body can lead to dose-dependent systemic side effects. ${ }^{24}$ Poor solubility of chemotherapeutic agents is another problem; low solubility makes it challenging to formulate a drug delivery system. Paclitaxel is an example of such a low solubility drug, which has limited application in chemotherapy. Low water solubility can lead to embolization of blood vessels when administered through the intravenous route. ${ }^{25,26}$ Furthermore, the tumor cells also develop resistance to chemotherapeutic agents. The interstitial fluids of tumor cells develop high hydrostatic pressure, leading to an outward convective interstitial flow that can remove the therapeutic agents from the tumor cells. ${ }^{27,28}$

Conventional drug delivery systems for the chemotherapeutic agents come across hurdles in the course of drugs' passage to the malignant tumor. The drug's physicochemical properties, comprising the surface composition, particle size, particle charges, demonstrate a substantial role in drug transportation to the site of action. ${ }^{29}$ Additional steeplechases involve the pathophysiological tumor heterogeneity, which constrains an unvarying drug delivery into the whole tumor mass. Acidic tumor microenvironment causes dilapidation of the acid-sensitive drugs.

\section{Various Types of Nanocarrier-Based Drug Delivery Systems}

If at all possible, for anticancer drugs to be significantly effective, they should initially, be capable of breaching over the obstructions in the body and targeting the anticipated tumor tissues with insignificant forfeiture of their volume or activity in the blood circulation. Subsequently, after attaining the target site, chemotherapeutic agents should have the capacity to eradicate the tumor cells without distressing healthy cells. These two rudimentary tactics are also connected with enhancements in patient endurance by increasing the accumulation of chemotherapeutic agents inside the tumor microenvironment and dropping dose-limiting toxicities all together. Progressively, nanocarriers give the impression to have the prospective to propitiate both of these necessities for effective drug carrier systems.

Various novel nanocarrier-mediated drug delivery systems to deliver the chemotherapeutic agents at targeted sites are currently in practice. The structural representation of different nanocarriers-based delivery systems is illustrated in Figure $1 .^{30}$ Some important nanocarriers include polymeric nanoparticles, liposomes, polymeric micelles, carbon nanotubes, dendrimers, solid lipid nanoparticles,

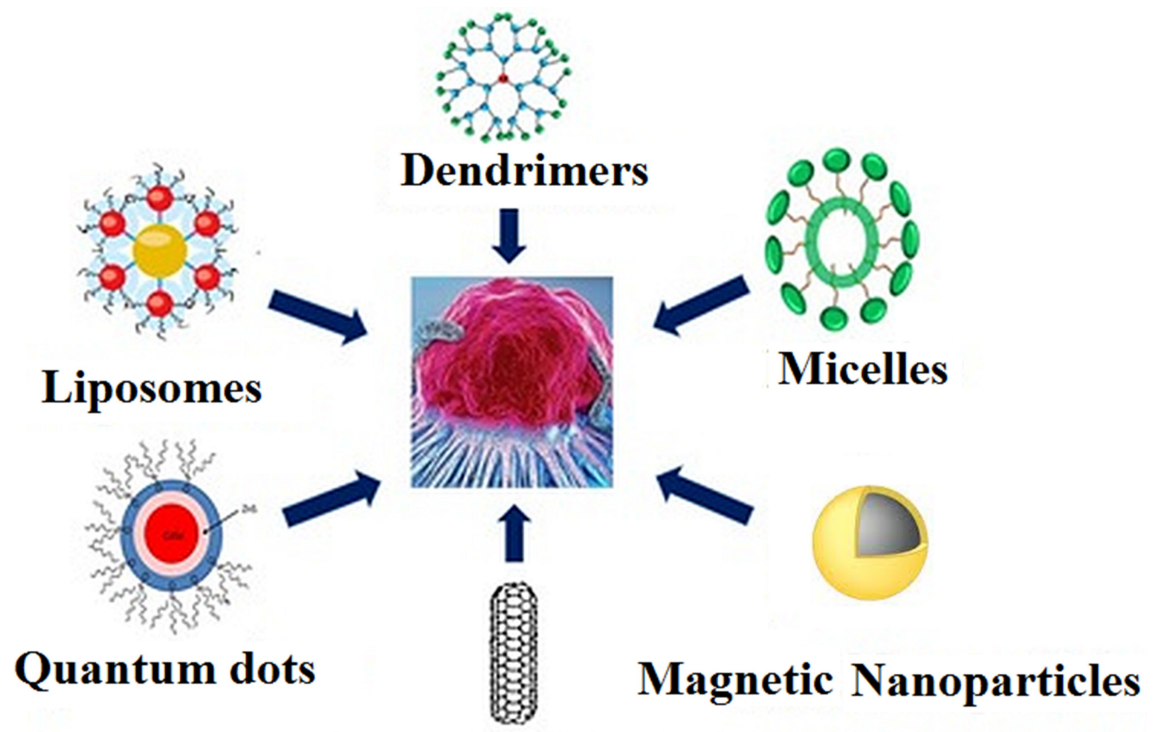

Carbon Nanotubes

Figure I Various types of nanocarriers used to treat the malignant tumors. 
magnetic nanoparticles, and quantum dots. These nanocarriers are discussed in the following sub-sections.

\section{Nanocarriers Developed from Polymer}

Various types of polymers are being employed for the preparation of nanocarriers; the active therapeutic agents are either physically entangled with polymeric matrix or bounded covalently with polymers. ${ }^{31}$ The prepared polymeric systems could be like a capsule, amphiphilic, or complicated branched macromolecule. Both natural and synthetic polymers are used for the development of nanocarriers.

\section{Micelles-Based Delivery Systems}

Micelles are constructed with the amphiphilic block copolymers, and their well-designed properties are based on the nature of copolymers, which mount up to form a nano-sized core/shell construction in hydrophilic-buffered media. The hydrophobic cavity of micelles acts as a reservoir for lipophilic drug molecules and outer hydrophilic shell help the micelle stability in an aqueous environment, and these properties make micelles suitable for intravenous administration. ${ }^{32}$ Micelles can be enriched with drug molecules by using two methods, chemical covalent attachment method and physical encapsulation methods. ${ }^{33,34}$ Recently, the first polymeric micelles formulated with paclitaxel known as Genexol-PM, are cremophor-free polymeric micelle-formulated paclitaxel. Micelles formulation of paclitaxel has been undergone a clinical trial for advanced refractory malignancies. Another class of micelles being developed is the multifunctional polymeric micelles decorated with steering ligands, imaging and therapeutic agents, ${ }^{35}$ and will turn out to be conventional amid numerous models of the micellar preparation soon. Recently, a research group established the novel drug delivery system of ursolic acid-loaded polymeric micelles (UA-PMs). The results shown in Figure 2 depict that formulation UA-PMs considerably improved the cell-growth along with inhibiting cell-migration properties against the human hepatocellular carcinoma cells HepG2 while displayed no toxicity to both human normal liver cell line L-02 in vitro and the normal
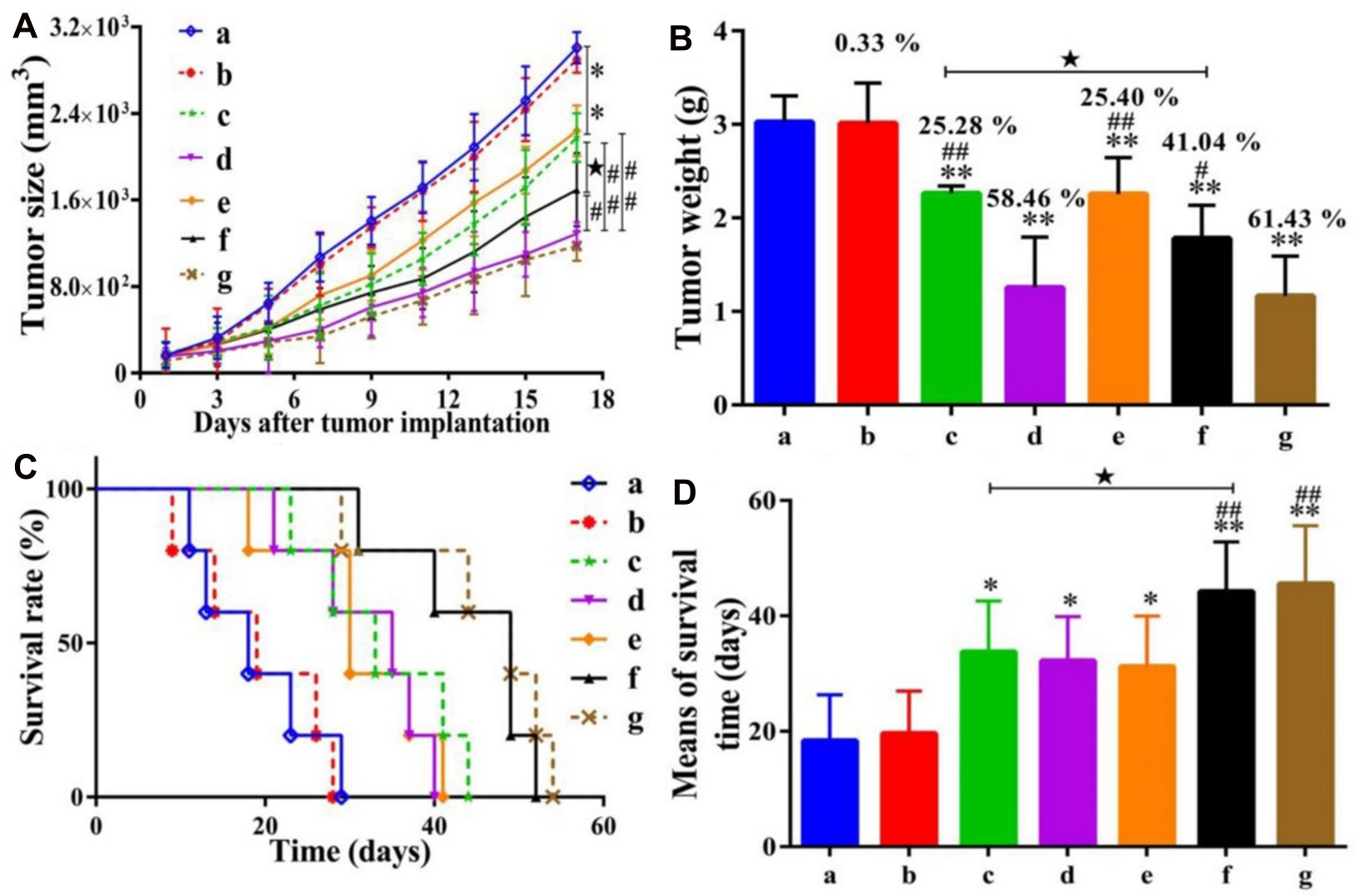

Figure 2 Determination of the antitumor activity of UA-PMs on $\mathrm{H}-22$ xenograft model. The mice induced with the $\mathrm{H}-22$ tumor were treated with different formulations. Outcomes measured the significant changes in the volume of tumor-induced in mice (A), changes in the weight of tumor (B), a curve showing the time of survival for the tumorbearing mice (C), mean of the survival time (D) and weight of mice. Data are shown as the mean \pm SD in each group $(n=5)$. Significant differences were observed between treated groups and saline $\left({ }^{* P}<0.05\right.$, $\left.{ }^{* * P}<0.01\right)$, treated groups and 5-FU group (\#P<0.05, \#\#P<0.0I), UA and UA-PMs ( $\left.{ }^{\star} \mathrm{P}<0.05\right)$ at the concentration of $50 \mathrm{mg} / \mathrm{kg}$. Adapted from Zhou M, Yi Y, Liu L, et al. Polymeric micelles loading with ursolic acid enhancing antitumor effect on hepatocellular carcinoma. J Cancer. 2019;10(23):5820. Creative Commons. ${ }^{36}$ 
Kunming mice in vivo. Additionally, the study revealed that UA-PMs could rescind the tumor development of H22 xenograft and lengthen the existence interval of the tumorbearing mice. ${ }^{36}$

\section{Nanoparticles Developed from Polymers}

Natural polymers like albumin, heparin and chitosan are used to create nanoparticles for the targeted transport of nucleotide-based drugs, oligonucleotide drugs and proteins. In recent times, nanometer-sized paclitaxel-loaded nanoparticles have been trialed in clinics for the treatment of metastatic mammary gland tumors. ${ }^{37}$ In addition to breast tumor-related, these nanoparticles have been evaluated for the management of other types of malignancies together with non-small-cell lung cancer are at Phase II trial, progressive non-haematologic malignancy trials are at Phase I and pharmacokinetic trials. ${ }^{38}$

A group of researchers has developed polymeric nanoparticle-based delivery systems with ultra-high drug loading glutathione sensitive heterodimeric multifunctional prodrug. The significantly fascinating property of the prodrug-loaded nanoparticles is enabling the quantitative pharmacoimaging, which helps in monitoring the distribution of drug within the tumor cells by PET imaging. As illustrated in Figure 3A-D, polymeric nanoparticles loaded with prodrug and isotopes showed significantly improved drug distribution within tumor. PET imaging confirmed significantly higher quantities of the drug for $28 \mathrm{hrs}$. As compared with HPPH, the momentous tumor accumulation of CPT-ssHPPH was verified by ex vivo bio-distribution evaluated via $\gamma$-counting of excised organs (Figure 3C). ${ }^{39}$

\section{Lipid-Based Drug Carriers}

Among the lipid-based nanoparticulate formulations, liposomes were initially designed in 1965; liposomes were the foremost nanocarriers employed in nanomedicine field. ${ }^{40}$ Nowadays, many liposomes based formulations are approved for clinical applications, and hundreds are in clinical and preclinical developments. The liposomes are superb drug delivery systems due to their easy degradation inside the body and additionally their property to encapsulate hydrophilic agents inside the aqueous environment and lipophilic agents in outer lipid layers. Further, to enhance the stability of liposomes, polyethylene glycol (PEG) is coated on the surfaces of liposomes. ${ }^{41}$ Pegylated liposomes demonstrated the reduction in the distribution volume of dox from 1000 $\mathrm{L} / \mathrm{m}^{2}$ in the free drug form to $2.8 \mathrm{~L} / \mathrm{m}^{2}$ by limiting the dissemination inside the plasma. Likewise, liposomal drug delivery limits the highest drug concentrations within the tumor cells and reduce the pooling of drug in other tissues. ${ }^{42}$ At present, quite a lot of antitumor agents are being formulated within these novel lipids based drug delivery systems with different methods approaches. There are few essential liposomal delivery systems loaded with anticancer agents like doxorubicin (Doxil, Myocet) and daunorubicin (DaunoXome). Both have been approved for the treatment of mammary gland tumors and AIDS-linked Kaposi's melanoma. ${ }^{43}$ In addition to these approved formulations, numerous antitumor formulations based on liposomes are at present being considered in clinical trials. The immunoliposomes seems to be the next generation of liposomal drugs, which selectively convey the drug to the anticipated sites of action. ${ }^{44}$

\section{Nanoparticles Developed from Solid Lipids}

Nanoparticles developed from solid lipids are typically spherical, nano-sized (10 to $1,000 \mathrm{~nm}$ ) colloidal carriers of natural or synthetic lipid-based drug delivery system. It is acclaimed that solid lipid nanoparticles are biocompatible and have a low toxicity profile. In contrast to polymeric or inorganic nanoparticles, lipid-based nanocarriers are less toxic and biocompatible. ${ }^{45,46}$ Solid lipid nanoparticles (SLNs) hold a solid core of the lipid matrix that can dissolve the lipophilic molecules. The term lipid is used generally in a vast sense and includes triglycerides, partial glycerides, PEGylated lipids, fatty acids, steroids, and waxes. ${ }^{47}$ The lipid core is balanced by using surfactants, as shown in Figure 4. The amount of emulsifier used depends on administration routes and is more limited for parenteral administrations. ${ }^{48,49}$ In this drug delivery system, small-sized lipid core, surfactants and drugs are present in appropriate ratios showing some unbeatable properties that make them superior over other existing drug carriers. These SLN-based preparations have some unique characteristics, including high drug loading, and increased drug stability. These formulations have better feasibility of hydrophilic and lipophilic drug incorporation. Generally, SLNs are highly compatible owing to their composition similarity from physiological lipids. Exceptional ability to incorporate drugs into nanocarriers and size-dependent properties of SLN makes them an ideal candidate to develop novel therapeutic prototypes for drug delivery and targeting. SLN has the potential to enhance the efficiency of pharmaceuticals, nutraceuticals, and other materials. In comparison to phospholipids and biodegradable polymers, solid lipids are economical. Compared to 
a)
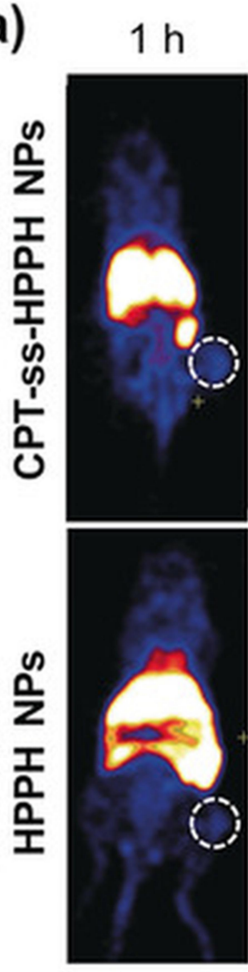

0
$6 \mathrm{~h}$

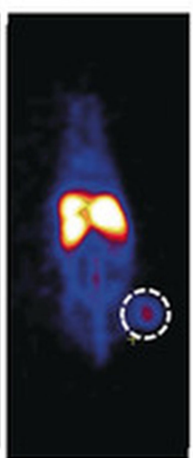

$24 \mathrm{~h}$
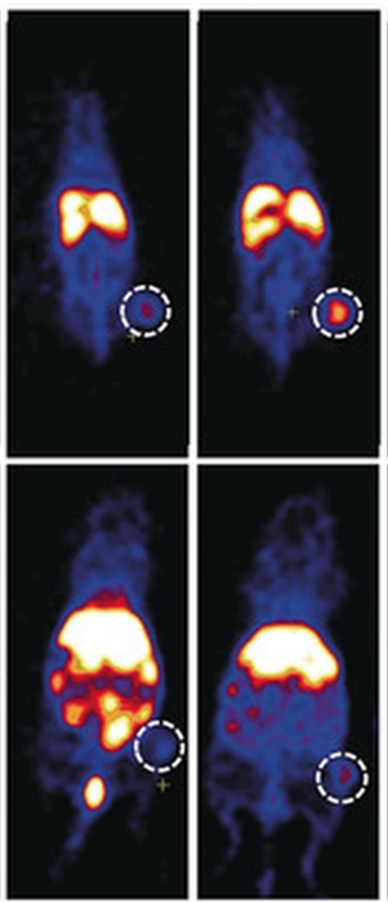

$48 \mathrm{~h}$
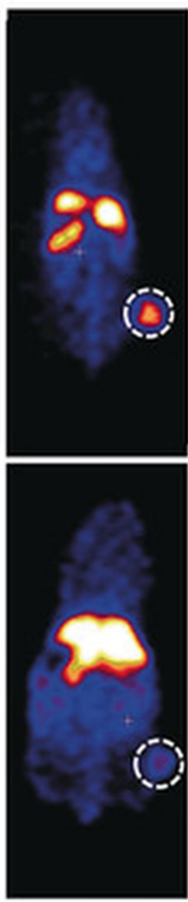

$10 \% \mathrm{ID} / \mathrm{g}$ b)

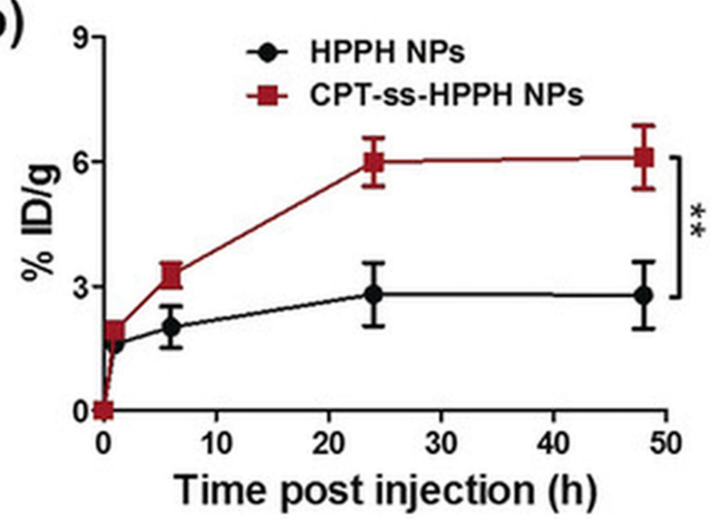

c)
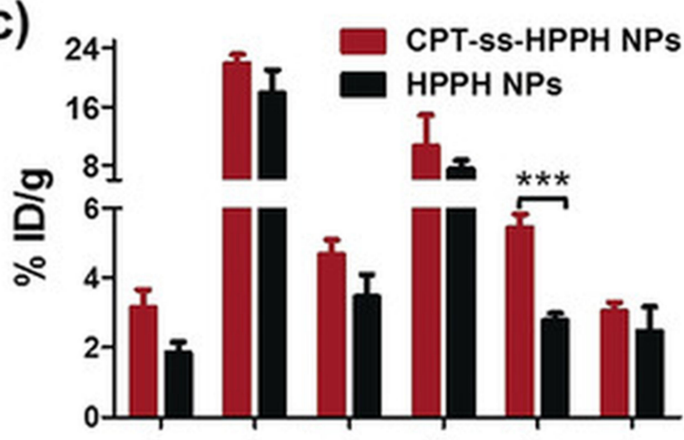

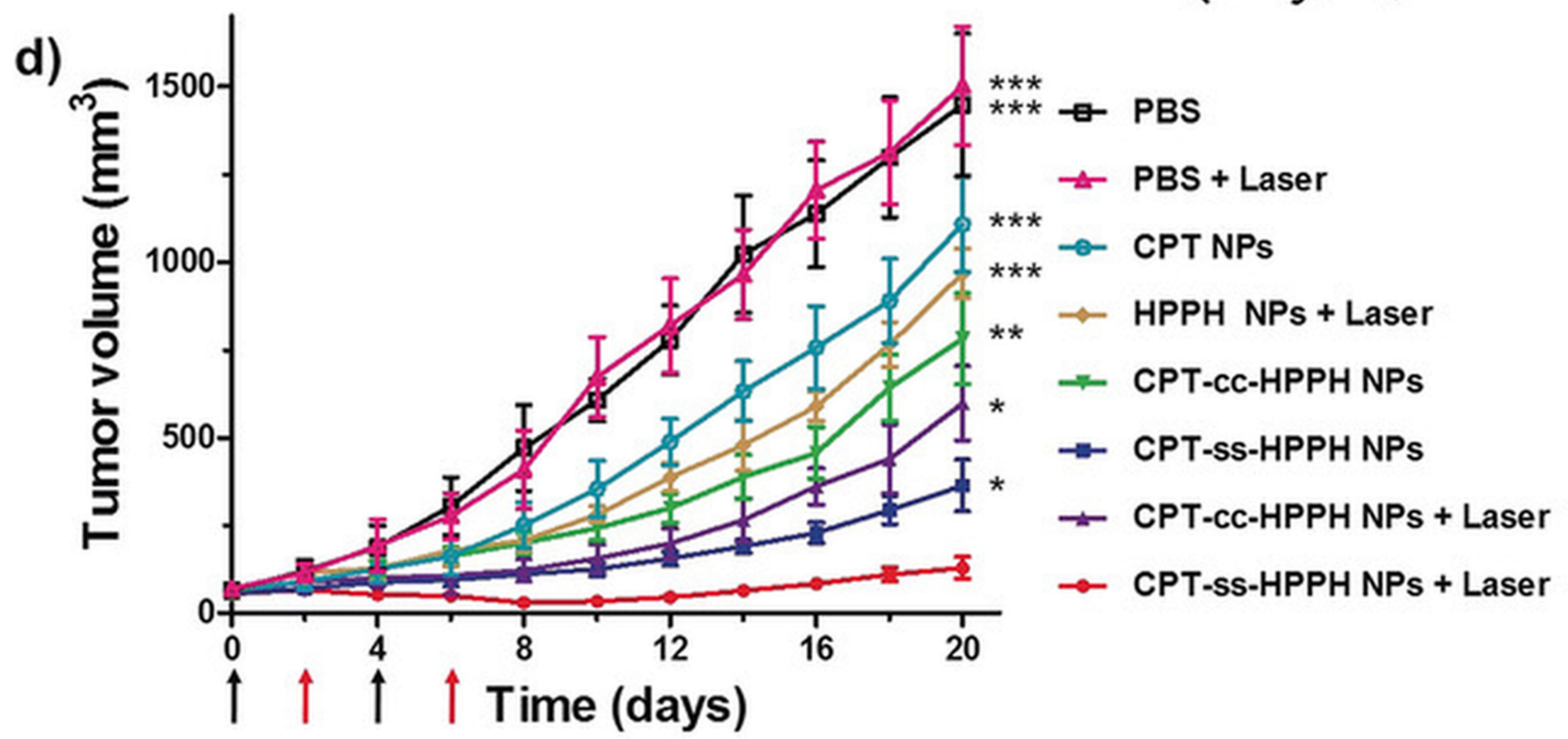

Figure 3 Heterodimeric multifunctional prodrug loaded NPs facilitated effective drug distribution to tumor and synergistic tumor chemotherapy and photodynamic therapy. (A) PET images showing the whole body of tumor-induced mice. Accumulation of NPs in tumors has been marked with white circles. (B) Quantification of the drug in tumor measured from decay-corrected PET images $(n=3)$. (C) Ex vivo drug dissemination quantified by $\gamma$-counting of excised tissues at $48 \mathrm{~h}$ after the injection $(\mathrm{n}=3)$. (D) The volume distribution of tumor under the influence of treatment $(n=5)$. Black arrow is a sign of intravenous injection of drugs, while red arrow reflects laser irradiation. Asterisk signs indicate the significant differences between CPT-ss-HPPH NPs and the other treatments. ${ }^{*} \mathrm{p}<0.05$; ${ }^{* *} \mathrm{p}<0.0 \mathrm{I}$; ${ }^{* * *} \mathrm{p}<0.00 \mathrm{I}$. ${ }^{*} \mathrm{p}<0.00 \mathrm{I}$. Adapted with permission from Zhang F, Ni Q, Jacobson O, et al. Polymeric nanoparticles with a glutathione-sensitive heterodimeric multifunctional prodrug for in vivo drug monitoring and synergistic cancer therapy. Angewandte Chemie Int Edition. 2018;57(24):7066-7070. @ 2018 Wiley-VCH Verlag GmbH \& Co. KGaA, Weinheim. ${ }^{39}$

traditional colloidal carriers, SLNs have superior cellular uptake and can improve the solubility and bioavailability of drugs. $^{50-53}$
Applications of SLN

In the recent past, SLN has emerged as a novel DDS for cancer research, including drug delivery, ${ }^{54}$ diagnosis, and 


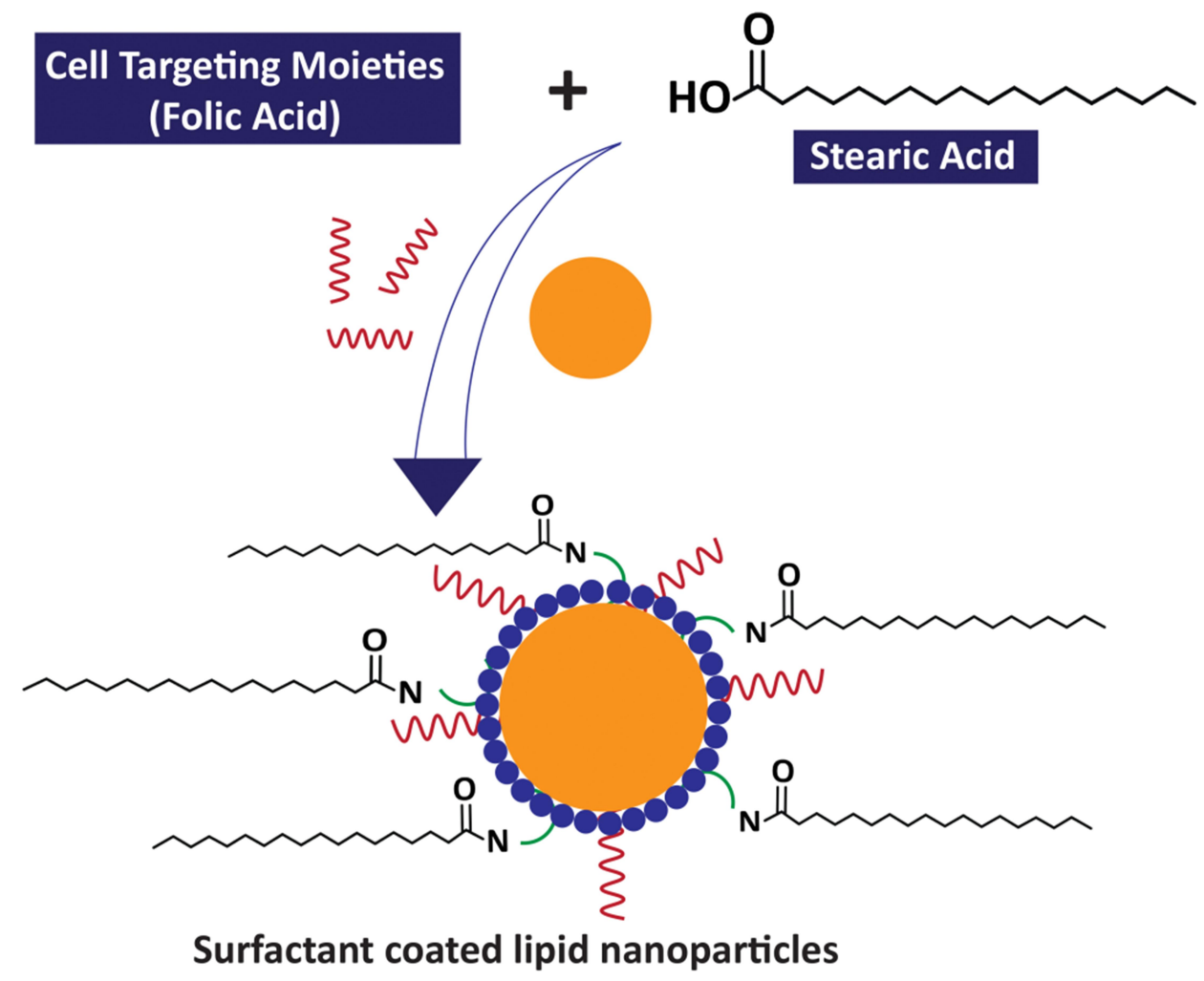

Figure $4 \mathrm{~A}$ schematic illustration of surfactant coating of solid lipid nanoparticles.

targeting. ${ }^{55,56} \mathrm{SLN}$, either single-handedly or in conjugation with other molecules, is extensively used in medicines, biomedical applications, bio-imaging, and photo-thermal therapy. ${ }^{57}$ Anticancer agents are delivered with improved selectivity, and activity is enhanced when delivered via novel SLNs. The SLNs-based delivery system also has the potential for cellular-based drug directing by altering cellular signaling and gene expression. ${ }^{58}$ Besides, SLNs are also deliberated as appropriate substitute carriers for parenteral delivery, ${ }^{59}$ gene delivery, ${ }^{60}$ pulmonary delivery, ${ }^{61}$ and topical and transdermal delivery. ${ }^{62,63}$ In this review, we will only discuss chemotherapeutic applications.

As a substitute to current conventional drug carriers (liposomes, colloidal systems, and polymeric and solid lipid nanoparticles), SLN has been developed as a new type of colloidal drug carrier system suitable for drug targeting applications. In the last decade, SLNs hold great potential for attaining the goal of targeted and controlled drug delivery, which currently draws the interest of researchers worldwide. SLN is a strategic vehicle for tumor targeting because of their passive targeting properties by the enhanced permeability and retention (EPR) effect. Stealth shielding is the additional advantage that avoids uptake by the reticuloendothelial system, thus prolonged the circulation time of the nanoparticles. ${ }^{64-66}$ Fundarò et al prepared doxorubicin-loaded stealth and nonstealth SLN by microemulsion template method to compare the pharmacokinetics and tissue distribution of doxorubicin-loaded SLN with commercially available doxorubicin solution. ${ }^{67}$ DOX-SLNs increased the concentration of DOX in the brain and CSF of rats, but low uptake of SLN by RES organs. Stealth SLN-based formulation showed an increase in half-life, AUC, and peak plasma concentration (7-fold). Up to 24 hours, prolonged circulation time was also achieved with an improved DOX formulation in DOX incorporated stealth SLNs. ${ }^{67}$

Sukumar et al reported the antitumor efficacy of SLNs by fabricating with variable proportions of stearic acid, lecithin, and phosphatidylserine. The SLN used in this work encapsulated phospho-sulindac was evaluated in human lung cancer xenograft models. ${ }^{68}$ Lung cancers are usually labeled as mutations in the p53 gene, resulting in loss of mutational repair and tumor-suppressor function, inhibition of apoptosis, increased tumor angiogenesis, cell proliferation, and increased drug resistance. ${ }^{69}$ SLNs are biodegradable, nonviral vectors that have gained increasing attention as 
promising colloidal carrier systems to treat early endobronchial cancer. SLN have shown their advantage over other carriers by their increased stability, no immunogenicity, and their controlled-release ability. ${ }^{70,71}$

Aiming at drug delivery, SLNs are developed to improve therapeutic delivery to target the cancerous cells by different mechanisms, including active targeting by external modification of SLNs, passive targeting leads to the malignant tumor microenvironment and provisions of co-delivery. ${ }^{72}$ SLNs can integrate various drug moieties and have recognized a suitable vehicle in different types of tumors (ie, lung, liver, breast, colon, and brain). SLNs have the potential to be used as a carrier for chemotherapeutic agents, corroborating their ability to improve pharmacokinetics and physicochemical stability of the integrated drugs. The lipoid nature and nano size of SLNs also influence the drug's pharmacokinetics through its specific uptake and distribution profile. ${ }^{73-76}$ In a study, nanoparticles developed via lipid hybridization loaded with cisplatin demonstrated higher uptake of the drug within the cell and significantly improved tumor growth inhibition in the ovarian cell line.

Additionally, pharmacokinetic and toxicity evaluation carried out in rabbits showed a controlled release behavior and provided these nanocarrier's safety profile. ${ }^{77}$ In contrast to other nanocarriers, SLN offers the possibility of a sustained-release due to its solid matrix. Owing to the smaller size and larger surface area, SLN-based DDS shows increase solubility and improved bioavailability. ${ }^{78,79}$ Furthermore, owing to the property of crossing the blood-brain barrier (BBB), SLN moved into the central nervous system and trapped by the cells more efficiently than larger molecules and, therefore, could be used as effective transport and delivery systems. ${ }^{80}$ Bartsch et al reported that SLNs could work as an efficient drug delivery system to deliver antisense oligonucleotide (ASODN) to liver endothelial cells in-vitro and in-vivo by stabilized lipid-coated lipoplexes. ${ }^{81}$

Today, researchers are continuously developing new nanocarriers to deliver therapeutic agents and imaging agents for cancer therapy and diagnosis, respectively. Exploring solid lipid nanocarriers promises the most exciting advances in lipid-based drug delivery to offer new hopes to address some of the most confounding illnesses for substantial signs of progress in cancer treatment and diagnosis. To date, several publications explained lipidbased carriers for lymphatic metastasis. In the course of lymph node metastasis, cancer cells first reach the SLN via lymph flow. For efficient SLN biopsy, cancer cells must be identified with high sensitivity in the SLN allied to the tumor site. In a study, ${ }^{82}$ Paliwal and colleagues developed SLNs for methotrexate delivery to the lymphatics. SLN being a lipid-based carrier retains a solid-state at physiological temperature. Results show a tenfold increase in lymphatic uptake after oral administration compared to methotrexate alone. ${ }^{82}$ In another study, Lu et al indicated that SLN of size $<100 \mathrm{~nm}$ is an ideal delivery system for chemotherapeutic agents against breast cancer and lymph node metastases. These SLN formulations showed sustained release with high drug targeting to the lymph nodes and a strong affinity to tumor tissue diagnosis. ${ }^{83}$

\section{Quantum Dots}

Nano-sized semiconductor quantum dots are among the novel strategies used in the treatment of different types of cancer. Water solubility and biocompatibility of quantum dots are improved by surface modification. It further helps the QDs to be used as fluorescent probes with targeting molecules. ${ }^{84}$ Quantum dot nanocarriers possess exceptional features like to have broad and robust absorption spectra and have unique optical and chemical properties. Semiconductor quantum dots can be used as photosensitizers. ${ }^{85,86}$ They show resilience to chemical degradation, $\mathrm{pH}$ changes, and have high thermal stability. QDs being an absorber of high-energy photons (X-rays and gamma rays) act as a radiosensitizer.

QDs are made up of a core and a shell or cap. ${ }^{87} \mathrm{~A}$ QD core includes various metal complexes, for example, noble metal, semiconductors, and magnetic transition metals. As, group III-V sequences QDs comprises of indium arsenate (InAs), indium phosphate (InP), gallium arsenate ( $\mathrm{GaAs})$, and gallium nitride $(\mathrm{GaN})$ metalloid cores, while group IIIV sequences QDs has cadmium-selenium (CdSe), zinc sulfide ( $\mathrm{ZnS})$, zinc-selenium ( $\mathrm{ZnSe}$ ) and cadmium- tellurium $(\mathrm{CdTe})$ cores. Cadmium contained semiconductors are the main component of quantum dots with noble optical characteristics. However, cadmium is probably harmful, and the toxicity studies of these cadmium-containing quantum dots in human living cells are comprehensively not evaluated yet. Consequently, exploration for non-toxic ingredients with comparable targeting and optical properties are of advanced concern.

\section{Applications of QDs}

QD-based nanocomposites have now become an exciting topic for research in the oncology field. QD nanomaterials 
are usually tiny in size, however, with wide-ranging application potentials in different fields. The excellent characteristics of carbon-based quantum dots, including low toxicity profile and biocompatibility empower remarkable biomolecule, drug delivery, bio-sensing, and bio-imaging applications.

High accuracy is required for effective cancer treatment through ionizing radiations to reduce exposure and toxicity to adjacent healthy cells. Some research has recently been dedicated to fabricating photosensitizerbased quantum dots that yield free radicals upon visible light immersion. Although visible light is harmless, the strategy is still appropriate to cure only superficial lumps. ${ }^{88,89}$ Various research invitations have revealed that QD-conjugated oligonucleotide sequences (attached through surface $\mathrm{COOH}$ groups) are targeted to connect with DNA or mRNA. ${ }^{90,91}$ Bio conjugated QDs are also considered for the site-specific gene as well as drug delivery in cancer. Targeting moieties, such as antibodies, ${ }^{92}$ high-molecular-weight dextran, ${ }^{93}$ aptamers, ${ }^{94}$ peptides, ${ }^{95}$ or folate, ${ }^{96}$ can then be combined with QDs.

Quantum dots, an emerging product of nanotechnology, can be used for biomedical and investigational imaging. Their extraordinary photo-physical characteristics and, at times, multifunctional faces are appropriate for several biological applications. QDs characterize the emerging application for cancer imaging with their unique and stable fluorescence, absorption and emission spectra and negligible photo-bleaching, and stable fluorescence. ${ }^{97,98}$

Although neurosurgery and radiotherapy have achieved significant advancement, yet the diagnosis of cancer patients with malignant gliomas has not shown any progress for the last decades. QDs show repetitious phases of excitation and fluorescence and a very narrow array of emission spectra. So the QDs are highly used in multiplexed imaging, in which several intensities and colors are united for small-molecules, genes coding protein libraries. For in-vivo studies, QDs are most widely used nowadays in sentinel lymph node mapping, localizing, and targeting tumors. As an alternative to radionuclide imaging procedures, optical imaging is now evolving as a new technique. The study's findings highlighted new paradigms and might be helpful for early diagnosis and therapy, including imaging-guided surgery. Researchers concluded that peptidelabeled NIR QD has the potential for in-vitro, ex-vivo tumor cell staining, and in-vivo tumor mass imaging. ${ }^{84,91}$

QDs have great potential for management as well as in the detection of cancers. Semiconductor quantum dots and nanocomposites made up of lipids, polymers, or metals have been developed with exciting cancer therapy applications and early detection. Presently only a few data are available regarding the application of QDs in whole-body imaging. QD-based multifunctional probes give excellent sensitivity for synchronized cancer molecular imaging and targeted therapy by their brightness. QD imaging technique has to turn into the most favorable tool for detecting cancer early due to its exceptional biomedical imaging performance. $^{99}$ Concurrent targeted DD and dual-mode tumor tissue imaging using near-infrared fluorescence and NMR spectroscopy. ${ }^{100}$ Gao and coworkers fabricated PEGylated quantum dots and then conjugated them with the anti-HER2 antibody and localized them in specific tumor cells. ${ }^{101}$

QDs have been encapsulated in lipid-based micelles for biological imaging applications. ${ }^{102}$ Lipid nanoparticles can simply be fabricated in bio-conjugation of several targeting moieties with a variety of functional groups $\left(\mathrm{SH}, \mathrm{NH}_{2}\right.$, biotin, $\mathrm{COOH}$, and maleimide) presented in phospholipids. ${ }^{103}$ The latest studies reported the preparation of lipodots; here, the QDs were incorporated in phospholipid vesicle bilayer. These folate-targeted lipodots are exploited for lymphoma cells targeting with overexpressed folate receptors, whereas colon carcinoma cells with not upregulated folate receptors did not respond to lipodots treatment. $^{104}$

Owing to their unique characteristics and properties, QDs are considered an efficient drug delivery system. QDs have the advantage of high specific surface area because multiple attachment sites are available for drug targeting; they can also easily cross the cell membranes. In this section potential of QDs as a delivery vehicle, connection with different ligands, and further improvements are also discussed. As an example, Olerile et al co-loaded chemotherapeutic drug paclitaxel (PTX) carrying SLN along with (CdTe/CdS/ZnS) QDs, and the results displayed higher drug loading and encapsulation efficacy with a tumor growth inhibition rate of 77.85\%. ${ }^{105}$ Another example is graphene QDs-based carboxymethyl cellulose hydrogel films were reported by Javanbakht and colleagues. ${ }^{23}$ In this work, nanocomposite hydrogel films were used as a delivery vehicle for doxorubicin (DOX). The results showed a sustained and pH-dependent release of DOX. ${ }^{106}$ Graphene-based QDS was developed as a DDS for the anticancer drug, methotrexate (MTX). GQDs were used as a cap with the polypyrrole mesoporous $(\mathrm{PPy} / \mathrm{mSiO} 2)$ core. PPy core is 
responsible for efficiently transforming near-infrared light into heat and acting as a photo-thermal agent. Nearinfrared light-triggered the drug release from the delivery vehicle by increasing the temperature of the system. Consequently, this increased temperature of nanocomposites leads to $\mathrm{H}$-bonds breakage between graphene QDs and $\mathrm{mSiO}_{2}$. GQDs also improved the encapsulation efficiency of MTX in the $\mathrm{PPy} / \mathrm{mSiO}_{2}{ }^{107}$

\section{Dendrimers}

Dendrimers are nanometer size drug delivery systems developed from the synthetic polymeric macromolecules, and dendrimers are constructed of manifold prominently branched monomers that project externally starting from the central core as shown in Figure 1. Dendrimer-based drug delivery systems possess a lot of unique properties since they have modifiable surfaces, monodisperse size, hydrophilic internal cavities and multivalences. ${ }^{108}$ Polyamidoamine dendrimers conjugated with cisplatin are famous for being used as scaffold systems. ${ }^{109}$ The only changeable external characteristic of dendrimers enables these unique systems to be concurrently conjugated with several molecules make them unique multifunctional drug delivery systems. ${ }^{110}$

\section{Applications of Dendrimers}

Amid the vast assortments of NPs available, dendrimers offer numerous benefits. Dendrimers because of their branched structures can encapsulate and conjugate particular type of drug molecules. A widespread variety of dendrimers used is the polyamidoamine (PAMAM) dendrimers, appropriate for numerous biomedical, biotechnological, regenerative medicine and tissue engineering applications. ${ }^{111}$ Polydiamine dendrimers are constructed of alkyl diamine inner core and peripheral branches of tertiary amine. ${ }^{112,113}$ The flexibility in the dendritic structure of dendrimers makes them perfect drug transporters in biomedical applications. ${ }^{114}$

As dendrimers hold a high density of functional surface molecules, they are straightforwardly conjugated with quite a lot of targeting agents for the pernickety supply of chemotherapeutics to the tumor tissue. Additionally, they encompass voids present in the core for encapsulation of lipophilic macromolecule, allowing them to transport highly hydrophobic drugs. ${ }^{115}$

Adding of stimuli response properties to the dendrimers make them versatile and release the drug loads in response to some specified triggers. These specified triggers can be internal from the tumor environment like acid, enzyme, and redox potentials or it can be induced from the external like light and temperature. ${ }^{116}$ In the latest work, integrated by Nigam et al for the treatment of cervical cancer, iron oxide NPs were merged with Generation 2 (G2) PAMAM dendrimers and encumbered with doxorubicin (DOX). Consequently, an amalgamation of the magnetic chemotherapy and hyperthermia was evaluated on HeLa cancer cells. ${ }^{117}$ When exposed to a flashing current magnetic field, the outcomes displayed improved cell death as a consequence of the lethal synergistic effect of DOX and high temperatures. Remarkably, the combinatorial treatment abridged the cancer cell viability from $100 \%$ to $3.6 \%{ }^{117}$

Another research group conjugated folic acid to PAMAM dendrimer NPs for targeting the tumor cells. ${ }^{118}$ The outcomes disclosed insignificant harmfulness to noncancerous MRC9 lung fibroblast cells, as well as the capability of dendrimer nanoparticles for targeted co-delivery of siRNA and chemotherapy agents together in the lung cancer cells. The dendrimers developed for targeting the breast tumor cells showed boosted in vitro cellular toxicity as compared with non-targeted dendrimers. The altered dendrimers displayed extraordinarily reduced relocation of BT-549-Luc breast cancer cells. Another research report confirmed the prospective utility of lactoferrin (LFC131) conjugated dendrimer for breast cancer therapy and metastasis. Galactosamine (Gal) is amongst the limited selective ligands used for targeting HCCs due to its high binding attraction to asialoglycoprotein receptors (ASGPRs) overexpressed in HCC. In the latest work, Yousef et al concocted a nanoscale G4 PAMAM dendrimer NP attached to Gal and laden with the potent anticancer curcumin derivative as a platform for targeted drug delivery to HCC. Astoundingly, in an in vivo xenograft model, cytotoxicity assays in HCC cell lines showed that curcumin derivative was more potent as a chemotherapeutic anticancer agent than the currently in use Doxorubicin, Sorafenib and Cisplatin chemotherapeutic agents. ${ }^{119}$ It has been pronounced that dendrimers have the magnetism to cross the blood-brain barrier after systemic administration. ${ }^{120}$ Liu et al utilized united chemo- and gene-therapy tactic for effective glioma treatment and established a DOX-loaded dendrigraft poly-L-lysine dendrimer surface modified with tumor necrosis factor associated apoptosis-inducing ligand (TRAIL) for the tumor-targeting explicitly of a specific peptide. $^{121}$

Colorectal cancer is the third maximum detected cancer in the world. Despite notable progress in treatment, 
CRC still leads to significant morbidity and mortality. ${ }^{122}$ Therefore, a research squad established nanotechnologybased cell recognition and apprehension technique. ${ }^{123}$ Refined anti-Slex (aSlex) antibody and FITC labeledsecond antibody (IgG/IgM-FITC) were employed for the amalgamation of aSlex-coated dendrimer conjugates. By exploiting this coating, dendrimers would internment to saliva acidifying Louis oligosaccharides X (Sialyl Lewis $\mathrm{X}$, Slex, a type II carbohydrate antigen for mediating the colorectal cancer metastatic process). By this technique, the colorectal cancer cells were not only captured in artificial blood samples but controlled in cell activity by the conjugates, which means, there was a specific interaction between antigen and antibody (existing in dendrimers).

Another study reported the incorporation of gold nanoparticles with dendrimers; the purpose of this research was to explore the theranostic competency of curcumin-loaded dendrimer-gold hybrid structures. The acquired consequences established higher cellular uptake, internalization and cytotoxicity of curcumin-loaded, gold nanoparticleloaded dendrimers in comparison with blank nanoparticles in C26 and HT29 colorectal cancer cell lines. Besides, the developed structure functioned as an effective antitumor therapy and accurate computed tomography imaging of C26 tumor-bearing mice due to gold accumulation. ${ }^{124}$

\section{Carbon Nanotubes}

Carbon nanotubes are mostly being applied biological fields as a sensor for diagnostic purpose and for detecting the DNA, proteins and discriminating different types of proteins from serum samples and help in the delivery of vaccines and proteins. ${ }^{125}$ These delivery systems pose some health dangers because of the insoluble nature in all kinds of solvents. Nevertheless, by the chemical changes in these tubes and attaching some functional groups make them water-soluble, and enable them to be used for delivery of lots of active therapeutics like, proteins, peptides, nucleic acids and other active moieties. Most interesting delivery systems have been developed by incorporating an antifungal agent (amphotericin B) and by combining the antitumor agents like methotrexate along with fluorescent agents. Research studies carried out with the drugs attached with nanotubes showed more effective internalization of drug molecules compared to free drug molecules. ${ }^{126}$ Similarly, human serum albumin has been attached with single-walled nanotubes for efficient loading of anticancerous compounds. The delivery systems showed significantly improved drug delivery inside the cells, up to $80 \%$ in breast cancer cells. The high binding capacity of PTX with HAS leads to the enhanced drug loading on SWNT-based drug delivery systems. These functionalized nanotubes displayed the most increased growth inhibition activity in MCF-7 breast cancer cells. ${ }^{127}$ The manifold covalent functionalization on the exteriors of carbon nanotubes permits them to convey more than a few drug molecules at once, and this approach offers a dynamic improvement in the management of cancer.

\section{Applications of Carbon Nanotubes}

Carbon nanotubes gain as mesmerizing nanocarriers for copious diagnostic and therapeutic applications for the reason that of elastic and conductive characteristics. ${ }^{128,129}$ CNTs generally exist in two forms: as Single-wall (SWCNTs) and Multi-Walled Carbon Nanotubes (MWCNTs) devouring benefits over globular nanoparticles for delivery applications. ${ }^{130}$ Carbon nanotubes with special characteristic badge advanced drug loading and imaging agents, which are amiable with multiple biological systems, confirmed by organized in vitro and in vivo studies. Platinum drugs can be conveyed via CNTs in three diverse modes: i) the drug can be loaded into the CNT cavity, ii) the drug attached directly to the CNT surface-functionalized with a carboxylic acid or amine groups, or iii) the drug can be linked through the use of a chemical tether. Furthermore, the internal and external planes of the CNTs were couturier with various functional groups, conjugates, targeted ligands or attaching PEG-like polymers to enhanced biocompatibility. ${ }^{131}$

Recently, Bianco et al $^{132}$ have emphasized the degradability of functionalized CNTs by oxidative enzymes. It suggests a new perception for its implication in the field of medical applications. The authors showed that monofunctionalisation, bi-functionalization, and tri-functionalization of CNTs provided high biocompatibility and translocated directly into the cytoplasm of cells. Non-degradable CNTs can be amassed in several organs and tissues. ${ }^{132}$ Furthermore, Allen et al efficaciously reconnoitred the degradation of pristine and oxidized SWCNTs with the help of oxidative enzyme horseradish peroxidase (HRP) as a catalytic agent. ${ }^{133}$ Lippard et al described earlier that $\mathrm{Pt}$ (IV) prodrug conjugated with soluble SWCNTs revealed successful delivery of CDDP using toxic dose on selective intracellular reduction. ${ }^{133}$ The same research group developed SWCNT-Pt (IV) complex with targeting molecules, containing a folate derivative (FA) at an axial 
position. The authors demonstrated the SWCNT-Pt (IV)FA efficacy over human cells, including choriocarcinoma and human nasopharyngeal carcinoma cells.

Moreover, Bhirde et al prepared the oxidized SWCNTs functionalized firstly with cisplatin followed by conjugation with epidermal growth factor (SWCNT-CDDP-EGF) for specific targeted drug delivery against squamous cancer cells. As a result, an increase in the EGF affinity towards cell surface receptor can selectively enter and kill the targeted cancer cells. Similarly, in-vitro studies were conducted on HNSCC cell lines (HN-13) using SWCNT-Qdot-EGF and SWCNT-Qdot as controls. Confocal microscopy and Qdot luminescence confirmed SWCNT-Qdot-EGF conjugates rapid internalized to HN13 cells, whereas negligible uptake happened for SWCNTQdot control due to low levels of EGFR. ${ }^{134}$ Hampel et al break new ground by filling up opened CNTs with Ptbased drug using wet chemical approach method where capillarity are the driving force for Carboplatin (CP) to enter the inner cavity. ${ }^{135}$ Drug loading can be altering according to change in temperature. However, just about $30 \%(\mathrm{w} / \mathrm{w})$ of CP was encumbered on the MWCNTs at the temperature of $90^{\circ} \mathrm{C}$. Moreover, $\mathrm{CP}$ inside MWCNTs was scrutinized using EDX, EELS, XPS and found platinum $\left(\mathrm{Pt}^{2+}\right)$ existing at oxidation state that point out the $\mathrm{CP}$ complex is very stable. ${ }^{136}$

\section{Magnetic Nanoparticles}

Magnetic particles are nanostructures of size ranges from 1-100 nm. MNPs usually contain a central magnetic core and a surface coati adjacent to the functional coat. Magnetic particles are assembled of pure metals such as iron, ${ }^{137}$ cobalt, ${ }^{137}$ nickel, ${ }^{138}$ and manganese, ${ }^{139}$ Mostly used in biomedical applications are iron oxide nanoparticles. ${ }^{140,141}$ Depending on magnetic characteristics, nanoparticles are classified as paramagnetic, ferromagnetic, diamagnetic, ferromagnetic, anti-ferromagnetic and super-paramagnetic materials. ${ }^{142}$ Diamagnetic materials are repelled in the magnetic field applied externally; thus, it results in zero magnetic moments in the absence of an external magnetic field. However, paramagnetic materials in the absence of a magnetic field do not retain their magnetic properties and thus forms a weak magnetic moment. Ferromagnetic materials will remain magnetized even when the external magnetic field is absent due to unpaired electrons. Their domains line up in magnetic field direction resulting in a massive magnetic moment. Antiferromagnetic materials result in zero magnetization because magnetic moments have opposite moments and equal magnitude. Ferri-magnetic materials have a net spontaneous magnetic moment because they consist of two different ions in which the opposing moments are unequal. Super-paramagnetism acts as a paramagnet in magnetic field presence ${ }^{143,144}$ Various magnetic nanoparticle features, like coating, size, and type of core material, all influence the biocompatibility or toxicity of magnetic nanoparticles. Ni and Co magnetic nanoparticles are susceptible to acid erosion and oxidation, and thus, they are considered toxic. In contrast, iron oxide nanoparticles are used extensively in biomedicine applications for several years because of their high chemical stability, mechanical hardness and magnetic properties in the radio frequency region. To enhance the efficacy of magnetic nanoparticles, various novel strategies of coating [with surfactants, inorganic materials (like silica, gold), polymers, and biomaterials] and functionalization are needed to enhance biocompatibility or to avoid phagocytosis by RES. ${ }^{143,145}$

In research work, iron oxide nanoparticles (IONP) loaded with doxorubicin were conjugated with recombinant human IGF1 as a targeting ligand. The influence of theranostic IONPs was assessed in a tissue-derived xenograft model, acquired from an orthotopic human pancreatic cancer patient. ${ }^{146}$ The outcomes of the study were that the nanoparticles based IGF1-IONPDOX formulation proficiently besieged to pancreatic tumors and were measurable by optical imaging and MRI, as displayed in Figure 5. Repetitive administration of IGF1-IONPDOX accomplished to break the barrier in the tumor stromal membrane that hinders the drug permeation, resulted in a reduction of tumor size and inhibited the growth of the human pancreatic tumor. ${ }^{147}$

\section{Applications of Magnetic Nanoparticles}

MNPs have paid attention from the last decades and appear to be an extremely suitable candidate for drug delivery. Applications of MNPs as a targeted drug delivery significantly rely on different aspects related to the size and magnetism of the biocompatible nanoparticles. ${ }^{144}$ MNPs can be used to overcome traditional DDS issues and deliver the API to the anticipated target area, sustaining the nanoparticles at a particular position during the drug release when joined with an exterior magnetic field. ${ }^{148}$ Consequently, MNPs, by binding with ligands, chemotherapeutics, or other drugs and antibodies, have been successfully used as drug carriers. Docetaxel is a lipophilic chemotherapeutic agent, which needs an 


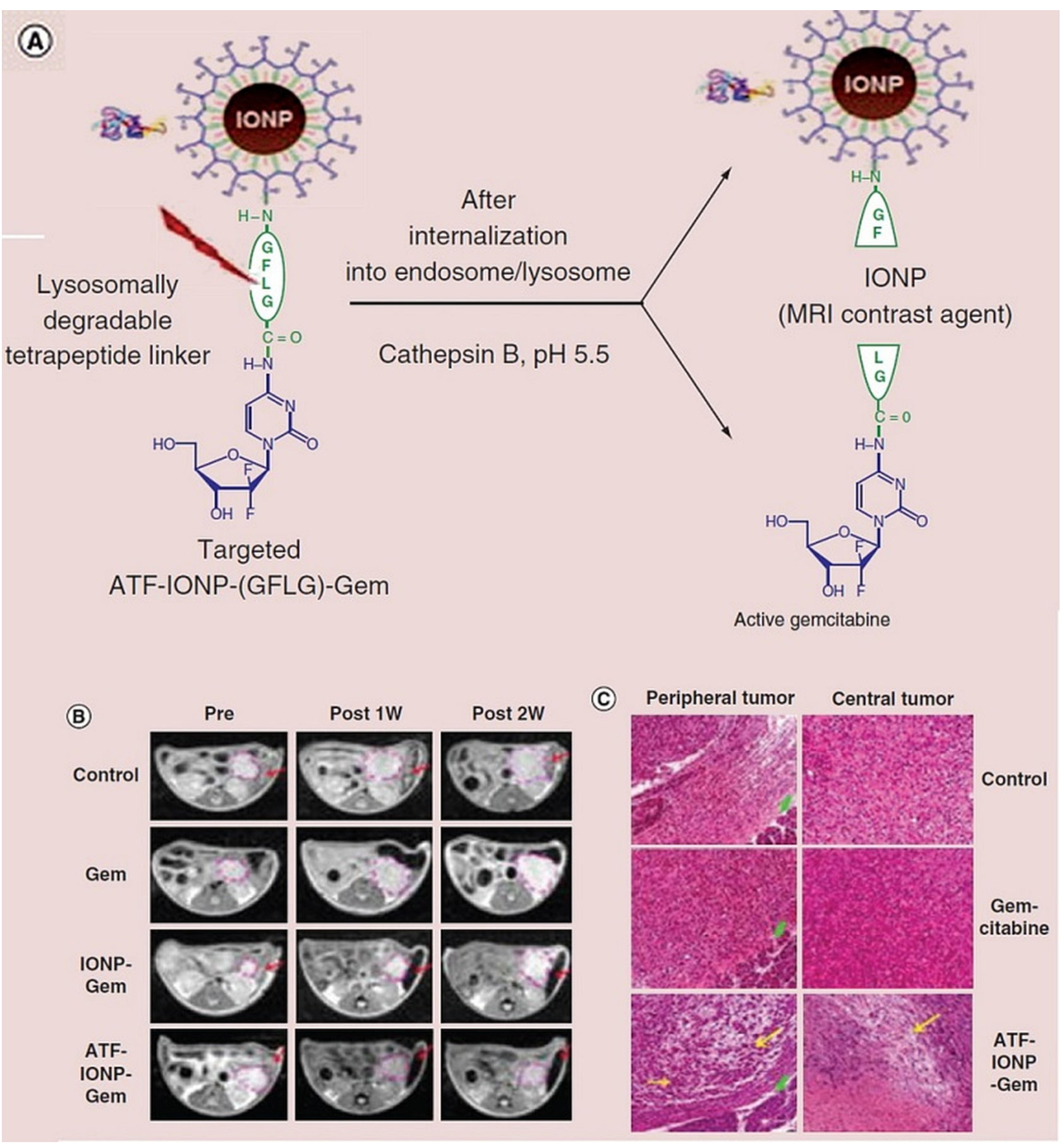

Figure 5 Magnetic resonance imaging of iron oxide nanoparticles based chemotherapy. (A) Scheme of development of gemcitabine conjugated iron oxide nanoparticles. (B) MRI observation of the therapeutic response of controlled released gemcitabine. (C) Histologic staining endorses the targeted therapeutic of pancreatic tumor. Adapted with permission from Lee GY, Qian WP, Wang L, et al. Theranostic nanoparticles with controlled release of gemcitabine for targeted therapy and MRI of pancreatic cancer. ACS Nano. 2013;7(3):2078-2089. Copyright (C) 2013, American Chemical Society. ${ }^{147}$

appropriate drug delivery system to increase its antitumor action. Iron oxide nanoparticles grafted with single-chain antibody and $\beta$-cyclodextrin for ovarian cancer dualtargeting therapy developed by Huang et al proved the pronounced perspective of combining chemotherapeutic agents with antibodies in the breast carcinoma. ${ }^{149}$ In addition, Aires et al fabricated a novel multifunctionalized iron oxide MNP by using the combination of antibodies and gemcitabine derivatives. ${ }^{150}$ A combination of anti CD44 antibody and chemotherapeutic drugs was used to select CD44-positive cancer cells to improve the therapeutic efficiency of gemcitabine. 
In medicine, magnetic nanoparticles are used as diagnostics and therapeutic tools. When used as a diagnostic agent, they possess both in-vivo and in-vitro applications like detection of various biomolecules, ${ }^{151}$ immobilization, purification, ${ }^{152}$ cell separation, ${ }^{153}$ and may serve as a contrast agent in MRI, ${ }^{154}$ or gene transfer. ${ }^{155}$ Magnetic nanoparticles provide skeletal or anatomical and metabolic/functional particulars with a high temporal and spatial resolution. ${ }^{156}$ It utilizes a high magnetic field to line up the nuclear magnetization of hydrogen atoms in the body to which later on radiofrequency. ${ }^{157}$ Pulse is put in that changes the lining up or alignment of nuclei. When the radiofrequency pulse is distant or removed, nuclei "relax" back to their original state. It provides three-dimensional high spatial resolutions and favorably contrasts differentiation between various soft tissues. Different contrast agents like MNPs and paramagnetic chelates were developed for MR imaging. ${ }^{158}$ The spatial resolution of $10-100 \mu \mathrm{m}$ with no imaging depth extremities is offered. ${ }^{159}$ MNPs are also used in photoacoustic imaging technique where they monitor the progressing diseases and tumors. In this technique, light energy is used as a source of excitation, and it ascertains all ultrasonic sound waves generated from the target. PA imaging explains subsurface tissue structures and functional changes with resolution up to 100 $\mu \mathrm{m} .{ }^{160}$ Optical imaging in-vivo depends on photons monitoring that was emitted from florescent agents in the near IR region. ${ }^{158}$ It has a capability to pass through various biological tissues. Fluorescent molecules absorb light at a particular wavelength and emit back the light at longer wavelengths. ${ }^{159}$ They were used in the molecular screening of different surface-based diseases.

\section{Conclusion}

Reviewing the significant complications of cancer concluded that a pivotal approach is needed for active treatment modalities for cancer. Traditional therapies, including chemotherapy, radiotherapy, and surgery, are still considered adequate, but due to specific side effects to the healthy body tissues, additional work for some advancement in cancer treatment modalities is the need of the hour. Nanocarriers-based approaches play a dynamic role in biomedical applications, particularly in drug delivery of chemotherapeutics. Versatile modifications are brought together to overcome conventional chemotherapy's limitations and reduce the toxicity in different nanocarriers. This review summarized the polymeric-based nanoparticles, magnetic nanoparticle, lipidbased carriers, dendrimers and quantum dots mediated drug delivery systems for anticancer agents that hold promising therapeutic outcomes. We also discussed the applications of these few important groups of nanocarriers in cancer drug delivery, drug targeting and cancer diagnosis. So based on the current review, it might be suggested that the nanocarriers developed from the healthy cells friendly biomaterials conjugated with tumor cells markers for the targeted and increased drug delivery at the site of action are better than the conventional nanocarrier systems.

\section{Future Perspectives}

However, there are still many challenges for nano-drug delivery systems to be successfully pragmatic for a suitable alternative to conventional chemotherapy for chemotherapeutics. The nanocarriers' toxicity, especially for QDs, is a significant obstacle for the development of a successful antitumor drug delivery system. Research is still going on to diminish the toxicity of existing nanocarriers and explore the advanced nanocarriers with a lower toxicity profile. Cadmium contained semiconductors are the main component of quantum dots with noble optical characteristics. However, cadmium is probably harmful and the toxicity studies of these cadmium containing quantum dots in human living cells are comprehensively not evaluated yet. Consequently, exploration for non-toxic ingredients with comparable targeting and optical properties are of advance concern.

Similarly, carbon nanotubes also hold promising future in the delivery of anticancer drugs, the manifold covalent functionalization on the exteriors of carbon nanotubes permits them to convey more than a few drug molecules at once, and this approach offers a dynamic improvement in the management of cancer.

\section{Acknowledgment}

This work was supported by the National Natural Science Foundation of China (81560461). This study was supported by medical high-level talent training plan and thousands of young and middle-aged backbone teachers' cultivation plan of Guangxi province.

\section{Disclosure}

The authors report no conflicts of interest in this work.

\section{References}

1. Ali I, Nadeem Lone M, Al-Othman Z, Al-Warthan A, Sanagi SM. Heterocyclic scaffolds: centrality in anticancer drug development. Curr Drug Targets. 2015;16(7):711-734. doi:10.2174/ 1389450116666150309115922 
2. Tsugane S, Sasazuki S. Diet and the risk of gastric cancer: review of epidemiological evidence. Gastric Cancer. 2007;10(2):75-83.

3. García-Aranda M, Immunotherapy: RM. A challenge of breast cancer treatment. Cancers. 2019;11(12):1822.

4. Arruebo M, Vilaboa N, Sáez-Gutierrez B, et al. Assessment of the evolution of cancer treatment therapies. Cancers. 2011;3 (3):3279-3330.

5. Aslam MS, Naveed S, Ahmed A, Abbas Z, Gull I, Athar MA. Side effects of chemotherapy in cancer patients and evaluation of patients opinion about starvation based differential chemotherapy. $J$ Cancer Ther. 2014;2014.

6. Rodgers GM, Becker PS, Blinder M, et al. Cancer-and chemotherapy-induced anemia. $J$ Nat ComprehensCancer Network. 2012;10(5):628-653.

7. Gharpure KM, Wu SY, Li C, Lopez-Berestein G, Sood AK. Nanotechnology: future of oncotherapy. Clin Cancer Res. 2015;21(14):3121-3130.

8. Jabir NR, Tabrez S, Ashraf GM, Shakil S, Damanhouri GA, Kamal MA. Nanotechnology-based approaches in anticancer research. Int J Nanomedicine. 2012;7:4391.

9. Patra JK, Das G, Fraceto LF, et al. Nano based drug delivery systems: recent developments and future prospects. J Nanobiotechnology. 2018;16(1):71.

10. Ali I, Nadeem Lone M, Suhail M, Danish Mukhtar S, Asnin L. Advances in nanocarriers for anticancer drugs delivery. Curr Med Chem. 2016;23(20):2159-2187. doi:10.2174/0929867323666160 405111152

11. Martinelli C, Pucci C, Ciofani G. Nanostructured carriers as innovative tools for cancer diagnosis and therapy. $A P L$ Bioengineering. 2019;3(1):011502.

12. Wolfram J, Zhu M, Yang Y, et al. Safety of nanoparticles in medicine. Curr Drug Targets. 2015;16(14):1671-1681.

13. Rizvi SA, Saleh AM. Applications of nanoparticle systems in drug delivery technology. Saudi Pharm J. 2018;26(1):64-70.

14. Stevanovic M, Uskokovic D. Poly (lactide-co-glycolide)-based micro and nanoparticles for the controlled drug delivery of vitamins. Curr Nanosci. 2009;5(1):1-14.

15. Zhang X, Li Y, Wei M, Liu C, Yu T, Yang J. Cetuximabmodified silica nanoparticle loaded with ICG for tumor-targeted combinational therapy of breast cancer. Drug Deliv. 2019;26(1):129-136.

16. Li M, Sun X, Zhang N, et al. NIR-Activated PolydopamineCoated Carrier-Free "Nanobomb" for In Situ On-Demand Drug Release. $A d v$ Sci. 2018;5(7):1800155.

17. Liu S, Pan J, Liu J, et al. Dynamically PEGylated and boratecoordination-polymer-coated polydopamine nanoparticles for synergetic tumor-targeted, chemo-photothermal combination therapy. Small. 2018;14(13):1703968.

18. Scherer F, Anton M, Schillinger U, et al. Magnetofection: enhancing and targeting gene delivery by magnetic force in vitro and in vivo. Gene Ther. 2002;9(2):102-109.

19. Alexiou $C$, Jurgons $R$, Schmid $R$, et al. In vitro and in vivo investigations of targeted chemotherapy with magnetic nanoparticles. J Magn Magn Mater. 2005;293(1):389-393.

20. Haacke EM, Cheng NY, House MJ, et al. Imaging iron stores in the brain using magnetic resonance imaging. Magn Reson Imaging. 2005;23(1):1-25.

21. Gleich B, Weizenecker J. Tomographic imaging using the nonlinear response of magnetic particles. Nature. 2005;435 (7046): 1214-1217.

22. Roduner E. Size matters: why nanomaterials are different. Chem Soc Rev. 2006;35(7):583-592.

23. Mays AN, Osheroff N, Xiao Y, et al. Evidence for direct involvement of epirubicin in the formation of chromosomal translocations in $\mathrm{t}(15 ; 17)$ therapy-related acute promyelocytic leukemia. Blood J Am Soc Hematology. 2010;115(2):326-330.
24. Deshpande PP, Biswas S, Torchilin VP. Current trends in the use of liposomes for tumor targeting. Nanomedicine. 2013;8(9):1509-1528.

25. Gao Y, Li Z, Xie X, et al. Dendrimeric anticancer prodrugs for targeted delivery of ursolic acid to folate receptor-expressing cancer cells: synthesis and biological evaluation. Eur J Pharm Sci. 2015;70:55-63.

26. Lipinski CA, Lombardo F, Dominy BW, Feeney PJ. Experimental and computational approaches to estimate solubility and permeability in drug discovery and development settings. Adv Drug Deliv Rev. 1997;23(1-3):3-25.

27. Szakács G, Paterson JK, Ludwig JA, Booth-Genthe C, Gottesman MM. Targeting multidrug resistance in cancer. Nat Rev Drug Discov. 2006;5(3):219-234.

28. Couvreur P, Vauthier C. Nanotechnology: intelligent design to treat complex disease. Pharm Res. 2006;23(7):1417-1450.

29. Park JH, Lee S, Kim J-H, Park K, Kim K, Kwon IC. Polymeric nanomedicine for cancer therapy. Prog Polym Sci. 2008;33(1):113-137.

30. Madni A, Batool A, Noreen S, et al. Novel nanoparticulate systems for lung cancer therapy: an updated review. $J$ Drug Target. 2017;25(6):499-512.

31. Rawat M, Singh D, Saraf S, Saraf S. Nanocarriers: promising vehicle for bioactive drugs. Biol Pharm Bull. 2006;29(9):1790-1798.

32. Adams ML, Lavasanifar A, Kwon GS. Amphiphilic block copolymers for drug delivery. J Pharm Sci. 2003;92(7):1343-1355.

33. Batrakova E, Dorodnych TY, Klinskii EY, et al. Anthracycline antibiotics non-covalently incorporated into the block copolymer micelles: in vivo evaluation of anticancer activity. $\mathrm{Br} J$ Cancer. 1996;74(10):1545-1552.

34. Nakanishi T, Fukushima S, Okamoto K, et al. Development of the polymer micelle carrier system for doxorubicin. $J$ Controlled Release. 2001;74(1-3):295-302.

35. Nasongkla N, Bey E, Ren J, et al. Multifunctional polymeric micelles as cancer-targeted, MRI-ultrasensitive drug delivery systems. Nano Lett. 2006;6(11):2427-2430.

36. Zhou M, Yi Y, Liu L, et al. Polymeric micelles loading with ursolic acid enhancing antitumor effect on hepatocellular carcinoma. J Cancer. 2019;10(23):5820.

37. Gradishar WJ, Tjulandin S, Davidson N, et al. Phase III trial of nanoparticle albumin-bound paclitaxel compared with polyethylated castor oil-based paclitaxel in women with breast cancer. J Clin Oncol. 2005;23(31):7794-7803.

38. Green M, Manikhas G, Orlov S, et al. Abraxane ${ }^{\circledR}$, a novel Cremophor ${ }^{\mathbb{B}}$-free, albumin-bound particle form of paclitaxel for the treatment of advanced non-small-cell lung cancer. Ann Oncol. 2006; 17(8):1263-1268.

39. Zhang F, Ni Q, Jacobson O, et al. Polymeric nanoparticles with a glutathione-sensitive heterodimeric multifunctional prodrug for in vivo drug monitoring and synergistic cancer therapy. Angewandte Chemie Int Edition. 2018;57(24):7066-7070.

40. Bangham A. Liposomes: the Babraham connection. Chem Phys Lipids. 1993;64(1-3):275-285.

41. Hare JI, Lammers T, Ashford MB, Puri S, Storm G, Barry ST. Challenges and strategies in anticancer nanomedicine development: an industry perspective. Adv Drug Deliv Rev. 2017;108:25-38.

42. Hofheinz R-D, Gnad-Vogt SU, Beyer U, Hochhaus A. Liposomal encapsulated anticancer drugs. Anticancer Drugs. 2005;16 (7):691-707.

43. Markman M. Pegylated liposomal doxorubicin in the treatment of cancers of the breast and ovary. Expert Opin Pharmacother. 2006;7(11):1469-1474.

44. Rosenthal E, Poizot-Martin I, Saint-Marc T, Spano J. Phase IV study of liposomal daunorubicin (DaunoXome) in AIDS-related Kaposi sarcoma. Am J Clin Oncol. 2002;25(1):57-59.

45. Attama AA, Momoh MA, Builders PF. Lipid nanoparticulate drug delivery systems: a revolution in dosage form design and development. Recent Adv Novel Drug Carrier Sys. 2012;5:107-140. 
46. Haider M, Abdin SM, Kamal L, Orive G. Nanostructured lipid carriers for delivery of chemotherapeutics: A review. Pharmaceutics. 2020;12(3):288.

47. Severino P, Andreani T, Macedo AS, et al. Current state-of-art and new trends on lipid nanoparticles (SLN and NLC) for oral drug delivery. J Drug Deliv. 2012;2012.

48. Khatak S, Dureja H. Structural Composition of Solid Lipid Nanoparticles for Invasive and Non-invasive Drug Delivery. Curr Nanomater. 2017;2(3):129-153.

49. Mishra DK, Dhote V, Bhatnagar P, Mishra PK. Engineering solid lipid nanoparticles for improved drug delivery: promises and challenges of translational research. Drug Deliv Transl Res. 2012;2(4):238-253.

50. Manjunath K, Reddy JS, Venkateswarlu V. Solid lipid nanoparticles as drug delivery systems. Methods Find Exp Clin Pharmacol. 2005;27(2):127-144.

51. Sathali A, Ekambaram P, Priyanka K. Solid lipid nanoparticles: a review. Sci Rev Chem Commun. 2012;2(1):80-102.

52. Yadav N, Khatak S, Sara US. Solid lipid nanoparticles-a review. Int J Appl Pharm. 2013;5(2):8-18.

53. Lingayat VJ, Zarekar NS, Shendge RS. Solid lipid nanoparticles: a review. Nanosci Nanotech Res. 2017;2:67-72.

54. Kumar CS, Thangam R, Mary SA, Kannan PR, Arun G, Madhan B. Targeted delivery and apoptosis induction of trans-resveratrol-ferulic acid loaded chitosan coated folic acid conjugate solid lipid nanoparticles in colon cancer cells. Carbohydr Polym. 2020;231:115682.

55. Bayón-Cordero L, Alkorta I, Arana L. Application of solid lipid nanoparticles to improve the efficiency of anticancer drugs. Nanomaterials. 2019;9(3):474.

56. Dolatabadi JEN, Valizadeh H, Hamishehkar H. Solid lipid nanoparticles as efficient drug and gene delivery systems: recent breakthroughs. Adv Pharm Bulletin. 2015;5(2):151.

57. Puri A, Loomis K, Smith B, et al. Lipid-based nanoparticles as pharmaceutical drug carriers: from concepts to clinic. Critical Rev Therapeutic Drug Carrier Sys. 2009;26:6.

58. Swathi G, Prasanthi N, Manikiran S, Ramarao N. Solid lipid nanoparticles: colloidal carrier systems for drug delivery. ChemInform. 2012;43(2).

59. Wissing S, Kayser O, Müller R. Solid lipid nanoparticles for parenteral drug delivery. Adv Drug Deliv Rev. 2004;56(9):1257-1272.

60. Karagöz U, Kotmakçı M, Akbaba H, Çetintaş VB, Kantarcı G. Preparation and characterization of non-viral gene delivery systems with pEGFP-C1 Plasmid DNA. Br J Pharm Sci. 2018;54:1.

61. Zhao Y, Chang Y-X, Hu X, Liu C-Y, Quan L-H, Liao Y-H. Solid lipid nanoparticles for sustained pulmonary delivery of Yuxingcao essential oil: preparation, characterization and in vivo evaluation. Int J Pharm. 2017;516(1-2):364-371.

62. Souto EB, Baldim I, Oliveira WP, et al. SLN and NLC for topical, dermal, and transdermal drug delivery. Expert Opin Drug Deliv. 2020;17(3):357-377.

63. Benson HA, Grice JE, Mohammed Y, Namjoshi S, Roberts MS. Topical and transdermal drug delivery: from simple potions to smart technologies. Curr Drug Deliv. 2019;16(5):444- 460.

64. Yang T, Cui F-D, Choi M-K, et al. Enhanced solubility and stability of PEGylated liposomal paclitaxel: in vitro and in vivo evaluation. Int J Pharm. 2007;338(1-2):317-326.

65. Béduneau A, Saulnier P, Benoit J-P. Active targeting of brain tumors using nanocarriers. Biomaterials. 2007;28(33):4947-4967.

66. Byrne JD, Betancourt T, Brannon-Peppas L. Active targeting schemes for nanoparticle systems in cancer therapeutics. $A d v$ Drug Deliv Rev. 2008;60(15):1615-1626.

67. Fundarò A, Cavalli R, Bargoni A, Vighetto D, Zara GP, Gasco MR. Non-stealth and stealth solid lipid nanoparticles (SLN) carrying doxorubicin: pharmacokinetics and tissue distribution after iv administration to rats. Pharmacol Res. 2000;42 (4):337-343
68. Sukumar UK, Bhushan B, Dubey P, Matai I, Sachdev A, Packirisamy G. Emerging applications of nanoparticles for lung cancer diagnosis and therapy. Int Nano Lett. 2013;3(1):45.

69. Nemunaitis J, Swisher SG, Timmons T, et al. Adenovirusmediated p53 gene transfer in sequence with cisplatin to tumors of patients with non-small-cell lung cancer. J Clin Oncol. 2000;18(3):609.

70. Mundargi RC, Babu VR, Rangaswamy V, Patel P, Aminabhavi TM. Nano/micro technologies for delivering macromolecular therapeutics using poly (D, L-lactide-co-glycolide) and its derivatives. J Controlled Release. 2008;125(3):193-209.

71. Singh M, Briones M, Ott G, O’Hagan D. Cationic microparticles: a potent delivery system for DNA vaccines. Proce Nat Acad Sci. 2000;97(2):811-816.

72. Kim CH, Lee SG, Kang MJ, Lee S, Choi YW. Surface modification of lipid-based nanocarriers for cancer cell-specific drug targeting. J Pharm Inves. 2017;47(3):203-227.

73. Din F, Aman W, Ullah I, et al. Effective use of nanocarriers as drug delivery systems for the treatment of selected tumors. Int J Nanomedicine. 2017;12:7291.

74. Arias Clares L. Lipid-based drug delivery systems for cancer treatment. Curr Drug Targets. 2011;12(8):1151-1165.

75. Lin Y-S, Lee M-Y, Yang C-H, Huang K-S. Active targeted drug delivery for microbes using nanocarriers. Curr Top Med Chem. 2015;15(15):1525-1531.

76. Attia MF, Anton N, Wallyn J, Omran Z, Vandamme TF. An overview of active and passive targeting strategies to improve the nanocarriers efficiency to tumour sites. J Pharm Pharm. 2019;71(8):1185-1198.

77. Khan MM, Madni A, Torchilin V, et al. Lipid-chitosan hybrid nanoparticles for controlled delivery of cisplatin. Drug Deliv. 2019;26(1):765-772.

78. Harms M, Müller-Goymann C. Solid lipid nanoparticles for drug delivery. J Drug Deliv Sci Technol. 2011;21(1):89-99.

79. Das S, Das MK. Surface Modification of Resorcinarene-Based Self-Assembled Solid Lipid Nanoparticles for Drug Targeting. Springer: Surface Modification of Nanoparticles for Targeted Drug Delivery; 2019:311-329.

80. Song H, Wei M, Zhang N, et al. Enhanced permeability of bloodbrain barrier and targeting function of brain via borneol-modified chemically solid lipid nanoparticle. Int $J$ Nanomedicine. 2018;13:1869.

81. Bartsch M, Weeke-Klimp AH, Hoenselaar EP, et al. Stabilized lipid coated lipoplexes for the delivery of antisense oligonucleotides to liver endothelial cells in vitro and in vivo. $J$ Drug Target. 2004;12(9-10):613-621.

82. Paliwal R, Rai S, Vaidya B, et al. Effect of lipid core material on characteristics of solid lipid nanoparticles designed for oral lymphatic delivery. Nanomedicine. 2009;5(2):184-191.

83. Lu B, Xiong S-B, Yang H, Yin X-D, Chao R-B. Solid lipid nanoparticles of mitoxantrone for local injection against breast cancer and its lymph node metastases. Eur j Pharm Sci. 2006;28 (1-2):86-95.

84. Cai W, Shin D-W, Chen K, et al. Peptide-labeled near-infrared quantum dots for imaging tumor vasculature in living subjects. Nano Lett. 2006;6(4):669-676.

85. Yaghini E, Seifalian AM, MacRobert AJ Quantum dots and their potential biomedical applications in photosensitization for photodynamic therapy. 2009.

86. Samia AC, Chen X, Burda C. Semiconductor quantum dots for photodynamic therapy. $J$ Am Chem Soc. 2003;125 (51):15736-15737.

87. Hardman R. A toxicologic review of quantum dots: toxicity depends on physicochemical and environmental factors. Environ Health Perspect. 2006;165-172. 
88. Samanta S, Kumar S, Battula V, Jaryal A, Sardana N, Kailasam K. Quantum dot-sensitized O-linked heptazine polymer photocatalyst for the metal-free visible light hydrogen generation. RSC Adv. 2020;10(50):29633-29641.

89. Cong S, Zhao Z Carbon Quantum Dots: A Component of Efficient Visible Light Photocatalysts: InTech; 2018.

90. Abbasi E, Kafshdooz T, Bakhtiary M, et al. Biomedical and biological applications of quantum dots. Artif Cells, Nanomed Biotechnol. 2016;44(3):885-891.

91. Jamieson $\mathrm{T}$, Bakhshi R, Petrova D, Pocock R, Imani M, Seifalian AM. Biological applications of quantum dots. Biomaterials. 2007;28(31):4717-4732.

92. Santana CP, Mansur AA, Carvalho SM. Bi-functional quantum dot-polysaccharide-antibody immunoconjugates for bioimaging and killing brain cancer cells in vitro. Mater Lett. 2019;252:333-337.

93. Wilson R, Spiller DG, Beckett A, Prior IA, Sée V. Highly stable dextran-coated quantum dots for biomolecular detection and cellular imaging. Chem Mater. 2010;22(23):6361-6369.

94. Wen L, Qiu L, Wu Y, Hu X, Zhang X. Aptamer-modified semiconductor quantum dots for biosensing applications. Sensors. 2017;17(8):1736.

95. Thovhogi N, Sibuyi NRS, Onani MO, Meyer M, Madiehe AM. Peptide-functionalized quantum dots for potential applications in the imaging and treatment of obesity. Int J Nanomedicine. 2018;13:2551.

96. Pal S, Dalal C, Jana NR. Supramolecular Host-Guest ChemistryBased Folate/Riboflavin Functionalization and Cancer Cell Labeling of Nanoparticles. ACS Omega. 2017;2(12):8948-8958.

97. Malik P, Gulia S, Kakkar R. Quantum dots for diagnosis of cancers. Adv Mat Lett. 2013;4:811-822.

98. Resch-Genger U, Grabolle M, Cavaliere-Jaricot S, Nitschke R, Nann T. Quantum dots versus organic dyes as fluorescent labels. Nat Methods. 2008;5(9):763.

99. Zhang H, Yee D, Wang C Quantum dots for cancer diagnosis and therapy: biological and clinical perspectives. 2008.

100. Park J-H, von Maltzahn G, Ruoslahti E, Bhatia SN, Sailor MJ. Micellar hybrid nanoparticles for simultaneous magnetofluorescent imaging and drug delivery. Angewandte Chemie. 2008;120 (38):7394-7398. doi:10.1002/ange.200801810

101. Gao J, Chen K, Miao Z, et al. Affibody-based nanoprobes for HER2-expressing cell and tumor imaging. Biomaterials. 2011;32 (8):2141-2148. doi:10.1016/j.biomaterials.2010.11.053

102. Dubertret B. In vivo imaging of quantum dots encapsulated in phospholipid micelles. Science. 2002;298(5599):1759-1762. doi:10.1126/science.1077194

103. Carion O, Mahler B, Pons T, Dubertret B. Synthesis, encapsulation, purification and coupling of single quantum dots in phospholipid micelles for their use in cellular and in vivo imaging. Nat Protoc. 2007;2(10):2383-2390. doi:10.1038/nprot.2007.351

104. Schroeder J, Shweky I, Shmeeda H, Banin U, Gabizon A. Folatemediated tumor cell uptake of quantum dots entrapped in lipid nanoparticles. J Controlled Release. 2007;124(1-2):28-34. doi:10.1016/j.jconrel.2007.08.028

105. Olerile LD, Liu Y, Zhang B, et al. Near-infrared mediated quantum dots and paclitaxel co-loaded nanostructured lipid carriers for cancer theragnostic. Colloids Surf B Biointerfaces. 2017;150:121-130. doi:10.1016/j.colsurfb.2016.11.032

106. Wei Z, Yin X, Cai Y, et al. Antitumor effect of a Pt-loaded nanocomposite based on graphene quantum dots combats hypoxia-induced chemoresistance of oral squamous cell carcinoma. <![CDATA[International Journal of Nanomedicine]] >. 2018;13:1505. doi:10.2147/IJN.S156984

107. Liu X, Shou D, Chen C, Mao H, Kong Y, Tao Y. Core-shell structured polypyrrole/mesoporous $\mathrm{SiO} 2$ nanocomposite capped with graphene quantum dots as gatekeeper for irradiation-controlled release of methotrexate. Mater Sci Eng. 2017;81:206-212. doi:10.1016/j.msec.2017.08.001
108. Li L, Wang J, Kong H, Zeng Y, Liu G. Functional biomimetic nanoparticles for drug delivery and theranostic applications in cancer treatment. Sci Tech Adv Materials. 2018;19(1):771-790. doi:10.1080/14686996.2018.1528850

109. Wang X, Wang Y, Chen ZG, Shin DM. Advances of cancer therapy by nanotechnology. Cancer Res Treatment. 2009;41(1):1.

110. Luo Y, Yin X, Yin X, et al. Dual $\mathrm{pH} /$ redox-responsive mixed polymeric micelles for anticancer drug delivery and controlled release. Pharmaceutics. 2019;11(4):176.

111. RVd A, SdS S, Igne Ferreira E, Giarolla J. New advances in general biomedical applications of PAMAM dendrimers. Molecules. 2018;23(11):2849.

112. Lee S, Son SJ, Song SJ, Ha TH, Choi JS. Polyamidoamine (PAMAM) dendrimers modified with cathepsin-B cleavable oligopeptides for enhanced gene delivery. Polymers. 2017;9 (6):224.

113. Tomalia DA, Fréchet JM. Discovery of dendrimers and dendritic polymers: a brief historical perspective. J Polym Sci a Polym Chem. 2002;40(16):2719-2728.

114. Oliveira JM, Salgado AJ, Sousa N, Mano JF, Reis RL. Dendrimers and derivatives as a potential therapeutic tool in regenerative medicine strategies-A review. Prog Polym Sci. 2010;35(9):1163-1194.

115. Sharma AK, Gothwal A, Kesharwani P, Alsaab H, Iyer AK, Gupta U. Dendrimer nanoarchitectures for cancer diagnosis and anticancer drug delivery. Drug Discov Today. 2017;22(2):314-326.

116. Gai S, Yang G, Yang $P$, et al. Recent advances in functional nanomaterials for light-triggered cancer therapy. Nano Today. 2018;19:146-187.

117. Nigam S, Bahadur D. Dendrimer-conjugated iron oxide nanoparticles as stimuli-responsive drug carriers for thermally-activated chemotherapy of cancer. Colloids Surf B Biointerfaces. 2017;155:182-192.

118. Amreddy N, Babu A, Panneerselvam J, et al. Chemo-biologic combinatorial drug delivery using folate receptor-targeted dendrimer nanoparticles for lung cancer treatment. Nanomedicine. 2018;14(2):373-384.

119. Yousef S, Alsaab HO, Sau S, Iyer AK. Development of asialoglycoprotein receptor directed nanoparticles for selective delivery of curcumin derivative to hepatocellular carcinoma. Heliyon. 2018;4(12):e01071.

120. Mishra V, Kesharwani P. Dendrimer technologies for brain tumor. Drug Discov Today. 2016;21(5):766-778.

121. Liu S, Guo Y, Huang R, et al. Gene and doxorubicin co-delivery system for targeting therapy of glioma. Biomaterials. 2012;33 (19):4907-4916.

122. Cisterna BA, Kamaly N, Choi WI, Tavakkoli A, Farokhzad OC, Vilos C. Targeted nanoparticles for colorectal cancer. Nanomedicine. 2016;11(18):2443-2456.

123. Xie J, Wang J, Chen H, et al. Multivalent conjugation of antibody to dendrimers for the enhanced capture and regulation on colon cancer cells. Sci Rep. 2015;5(1):1-10.

124. Gulbake A, Jain A, Jain A, Jain A, Jain SK. Insight to drug delivery aspects for colorectal cancer. World j Gastroenterology. 2016;22(2):582.

125. Zhao K, Shi N, Sa Z, Wang HX, Lu CH, Xu XY. Text mining and analysis of treatise on febrile diseases based on natural language processing. World J Tradit Chin Med. 2020;6:67-73.

126. Minelli C, Lowe SB, Stevens MM. Engineering nanocomposite materials for cancer therapy. Small. 2010;6(21):2336-2357.

127. Shao W, Paul A, Rodes L, Prakash S. A new carbon nanotube-based breast cancer drug delivery system: preparation and in vitro analysis using paclitaxel. Cell Biochem Biophys. 2015;71(3):1405-1414.

128. Boncel S, Zając P, Koziol KK. Liberation of drugs from multi-wall carbon nanotube carriers. $J$ Controlled Release. 2013;169(1-2):126-140. 
129. Eatemadi A, Daraee H, Karimkhanloo H, et al. Carbon nanotubes: properties, synthesis, purification, and medical applications. Nanoscale Res Lett. 2014;9(1):393.

130. Wong BS, Yoong SL, Jagusiak A, et al. Carbon nanotubes for delivery of small molecule drugs. Adv Drug Deliv Rev. 2013;65 (15):1964-2015.

131. Matsumura S, Ajima K, Yudasaka M, Iijima S, Shiba K. Dispersion of cisplatin-loaded carbon nanohorns with a conjugate comprised of an artificial peptide aptamer and polyethylene glycol. Mol Pharm. 2007;4(5):723-729.

132. Li J, Yap SQ, Yoong SL, et al. Carbon nanotube bottles for incorporation, release and enhanced cytotoxic effect of cisplatin. Carbon. 2012;50(4):1625-1634.

133. Gao L, Jia CH, Wang W. Recent advances in the study of ancient books on traditional Chinese medicine. World J Tradit Chin Med. 2020;6:61-66.

134. Bhirde AA, Patel V, Gavard J, et al. Targeted killing of cancer cells in vivo and in vitro with EGF-directed carbon nanotube-based drug delivery. ACS Nano. 2009;3(2):307-316.

135. Bhirde AA, Sousa AA, Patel V, et al. Imaging the distribution of individual platinum-based anticancer drug molecules attached to single-wall carbon nanotubes. Nanomedicine. 2009;4(7):763-772.

136. Tsang S, Chen Y, Harris P, Green M. A simple chemical method of opening and filling carbon nanotubes. Nature. 1994;372 (6502):159-162.

137. Smolensky ED, Park HYE, Berquó TS, Pierre VC. Surface functionalization of magnetic iron oxide nanoparticles for MRI applications-effect of anchoring group and ligand exchange protocol. Contrast Media Mol Imaging. 2011;6(4):189-199.

138. Kale SN, Jadhav AD, Verma S, et al. Characterization of biocompatible $\mathrm{NiCo} 2 \mathrm{O} 4$ nanoparticles for applications in hyperthermia and drug delivery. Nanomedicine. 2012;8(4):452-459.

139. Sayed FN, Jayakumar OD, Sudakar C, Naik R, Tyagi AK. Possible weak ferromagnetism in pure and $\mathrm{M}(\mathrm{Mn}, \mathrm{Cu}, \mathrm{Co}, \mathrm{Fe}$ and $\mathrm{Tb}$ ) doped NiGa2O4 nanoparticles. J Nanosci Nanotechnol. 2011;11(4):3363-3369.

140. Grassi-Schultheiss P, Heller F, Dobson J. Analysis of magnetic material in the human heart, spleen and liver. Biometals. 1997;10(4):351-355.

141. Banerjee IA, Yu L, Shima M, et al. Magnetic nanotube fabrication by using bacterial magnetic nanocrystals. Adv Mater. 2005;17 (9):1128-1131.

142. Yadollahpour A, Rashidi S. Magnetic nanoparticles: a review of chemical and physical characteristics important in medical applications. Oriental J Chem. 2015;31(Special Issue 1):25-30.

143. Neuberger T, Schöpf B, Hofmann H, Hofmann M, Von Rechenberg B. Superparamagnetic nanoparticles for biomedical applications: possibilities and limitations of a new drug delivery system. J Magn Magn Mater. 2005;293(1):483-496.

144. Akbarzadeh A, Samiei M, Davaran S. Magnetic nanoparticles: preparation, physical properties, and applications in biomedicine. Nanoscale Res Lett. 2012;7(1):144.
145. Chen KX. Academician kai-xian chen talks about the development of traditional chinese medicine and global medicine. World $J$ Tradit Chin Med. 2020;6:1-11.

146. Zhou H, Qian W, Uckun FM, et al. IGF1 receptor targeted theranostic nanoparticles for targeted and image-guided therapy of pancreatic cancer. ACS Nano. 2015;9(8):7976-7991.

147. Lee GY, Qian WP, Wang L, et al. Theranostic nanoparticles with controlled release of gemcitabine for targeted therapy and MRI of pancreatic cancer. ACS Nano. 2013;7(3):2078-2089.

148. Lima-Tenorio MK, Pineda EAG, Ahmad NM, Fessi H, Elaissari A. Magnetic nanoparticles: in vivo cancer diagnosis and therapy. Int J Pharm. 2015;493(1-2):313-327.

149. Huang X, Yi C, Fan Y, et al. Magnetic Fe3O4 nanoparticles grafted with single-chain antibody $(\mathrm{scFv})$ and docetaxel loaded $\beta$ cyclodextrin potential for ovarian cancer dual-targeting therapy. Mater Sci Eng. 2014;42:325-332.

150. Aires A, Ocampo SM, Simões BM, et al. Multifunctionalized iron oxide nanoparticles for selective drug delivery to CD44-positive cancer cells. Nanotechnology. 2016;27(6):065103.

151. Tong HY, Zhang SQ, Murtaza G, et al. The present scenario, challenges, and future anticipation of traditional Mongolian medicine in China. World J Tradit Chin Med. 2019;4:187-192.

152. Dong H, Huang J, Koepsel RR, Ye P, Russell AJ, Matyjaszewski K. Recyclable antibacterial magnetic nanoparticles grafted with quaternized poly (2-(dimethylamino) ethyl methacrylate) brushes. Biomacromolecules. 2011;12(4):1305-1311.

153. Huang Y-F, Wang Y-F, Yan X-P. Amine-functionalized magnetic nanoparticles for rapid capture and removal of bacterial pathogens. Environ Sci Technol. 2010;44(20):7908-7913.

154. Meng X, Seton HC, Lu LT, Prior IA, Thanh NT, Song B. Magnetic CoPt nanoparticles as MRI contrast agent for transplanted neural stem cells detection. Nanoscale. 2011;3(3):977-984.

155. Yiu HH, Pickard MR, Olariu CI, Williams SR, Chari DM, Rosseinsky MJ. Fe 3 O 4-PEI-RITC magnetic nanoparticles with imaging and gene transfer capability: development of a tool for neural cell transplantation therapies. Pharm Res. 2012;29(5):1328-1343.

156. Harisinghani MG, Barentsz J, Hahn PF, et al. Noninvasive detection of clinically occult lymph-node metastases in prostate cancer. New Eng J Med. 2003;348(25):2491-2499.

157. Yang SJ, Wang ZY, Zhao HH, Ren XQ. Modern research of tibetan medicine. World J Tradit Chin Med. 2019;5:131-138.

158. Frullano L, Meade TJ. Multimodal MRI contrast agents. JBIC J Biol Inorganic Chem. 2007;12(7):939-949.

159. Weissleder R, Pittet MJ. Imaging in the era of molecular oncology. Nature. 2008;452(7187):580-589.

160. Vallabani NV, Singh S, Karakoti AS. Magnetic nanoparticles: current trends and future aspects in diagnostics and nanomedicine. Curr Drug Metab. 2019;20(6):457-472.
International Journal of Nanomedicine

\section{Publish your work in this journal}

The International Journal of Nanomedicine is an international, peerreviewed journal focusing on the application of nanotechnology in diagnostics, therapeutics, and drug delivery systems throughout the biomedical field. This journal is indexed on PubMed Central, MedLine, CAS, SciSearch ${ }^{\mathbb{}}$, Current Contents ${ }^{\mathbb{R}} /$ Clinical Medicine,
Journal Citation Reports/Science Edition, EMBase, Scopus and the Elsevier Bibliographic databases. The manuscript management system is completely online and includes a very quick and fair peer-review system, which is all easy to use. Visit http://www.dovepress.com/ testimonials.php to read real quotes from published authors 